\title{
AN INNER MODELS PROOF OF THE KECHRIS-MARTIN THEOREM
}

\author{
ITAY NEEMAN
}

\begin{abstract}
We give an inner model theoretic proof of the Kechris-Martin theorem, stating under determinacy that the pointclass $\Pi_{3}^{1}$ is closed under existential quantifiers on ordinals below $u_{\omega}$, the $\omega$ th uniform indiscernible, and of related results, including a characterization of $\Pi_{3}^{1}$ using $\Sigma_{1}$ truth in least admissible structures over $T_{2}$, the MartinSolovay tree for $\Pi_{2}^{1}$. The inner model theoretic proofs rely on approximations for $T_{2}$ in models for one Woodin cardinals, and a correctness lemma for these approximations.
\end{abstract}

\$1. Introduction. The Kechris-Martin theorem is a fundamental result in the study of determinacy, stating that the pointclass $\Pi_{3}^{1}$ is closed under existential quantification over ordinals below $u_{\omega}$, the $\omega$ th uniform indiscernible, which under the full axiom of determinacy is equal to $\aleph_{\omega}$. There are by now several approaches to proving the theorem, that range from the purely descriptive set theoretic, to mixtures of descriptive set theory and inner model theory at the level of sharps for reals. This paper presents a different proof, that relies purely on inner model theoretic methods, at the levels of sharps for reals and sharps for one Woodin cardinal. The hope is that any additional methods for proving the theorem may help generalize it; generalizations of the theorem to higher levels of the projective hierarchy, with quantification over correspondingly greater ordinals of course, are still open.

To give a precise statement of the Kechris-Martin theorem we need to say exactly what we mean by quantification over ordinals below $u_{\omega}$. For this, the ordinals must be coded as reals. Let $u_{0}=\omega$ and let $u_{n}, 1 \leq n<\omega$, enumerate the first $\omega$ uniform indiscernibles in increasing order. (Under the full axiom of determinacy, $u_{n}=\omega_{n}$.) Define a code for an ordinal $\alpha \in\left[u_{n}, u_{n+1}\right)$ to be a pair $\left\langle x^{\sharp}, t\left(v_{1}, \ldots, v_{n}\right)\right\rangle$ so that $t^{L(x)}\left[u_{1}, \ldots, u_{n}\right]$ is equal to $\alpha . t\left(v_{1}, \ldots, v_{n}\right)$ here denotes a term in the language of set theory. Precisely it is a formula in variables $v_{1}, \ldots, v_{n}$ and $y$. In any model $M$ with a canonical wellordering of its elements, $t^{M}\left[a_{1}, \ldots, a_{n}\right]$ is the least $y$ so that the formula holds of $a_{1}, \ldots, a_{n}$ and $y$ if such $y$ exists, and undefined otherwise.

Given a code $w=\left\langle x^{\sharp}, t\left(v_{1}, \ldots, v_{n}\right)\right\rangle$, we write $|w|$ for the ordinal $\alpha$ that it codes. We use the letter $w$ to range over codes throughout this paper.

A set of ordinals $A \subseteq u_{\omega}$ belongs to the pointclass $\Gamma$ if the set $\{w|| w \mid \in A\}$ belongs to $\Gamma$. Note that since codes are elements of $\mathbb{R} \times \omega$, the question of whether $\{w|| w \mid \in A\}$ is in $\Gamma$ makes sense.

This material is based upon work supported by the National Science Foundation under Grant No. DMS-0556223 
An individual ordinal $\alpha<u_{\omega}$ belongs to the pointclass $\Gamma$ if it has a code $w$ in $\Gamma$. This concept has non-trivial meaning in case $\Gamma$ is a lightface pointclass.

We can now phrase the Kechris-Martin theorem precisely. The theorem itself is a consequence of a basis theorem for $\Pi_{3}^{1}$ sets of ordinals, which in turn is a consequence of a basis lemma for bounds on $\Sigma_{3}^{1}$ sets. All lemmas and theorems here are in $\mathrm{ZF}+\mathrm{DC}+\boldsymbol{\Delta}_{2}^{1}$ determinacy.

Lemma 1.1 (Kechris-Martin [1]). Let $A \subseteq u_{n+1}$ be $\Sigma_{3}^{1}$ and bounded below $u_{n+1}$. Then $A$ has a $\Delta_{3}^{1}$ bound.

THEOREM 1.2 (Kechris-Martin [1]). Every non-empty $\Pi_{3}^{1}$ set of ordinals below $u_{\omega}$ has a $\Delta_{3}^{1}$ member.

THEOREM 1.3 (Kechris-Martin [1]). $\Pi_{3}^{1}$ is closed under existential quantification over ordinals below $u_{\omega}$.

Theorem 1.3 is an immediate consequence of Theorem 1.2. Indeed, for every $n<\omega$, Theorem 1.3 for subsets of $u_{n+1}$ is an immediate consequence of Theorem 1.2 for subsets of $u_{n+1}$. Theorem 1.2 in turn can be proved using Lemma 1.1, as follows. Let $A \subseteq u_{\omega}$ be $\Pi_{3}^{1}$ and non-empty. Let $n<\omega$ be least so that $A \cap u_{n+1}$ is non-empty. Inductively we may assume that Theorem 1.2 holds for subsets of $u_{n}$, and hence so does Theorem 1.3. The set $B=\left\{\alpha<u_{n+1} \mid A\right.$ has no elements below $\left.\alpha\right\}$ is bounded. From any code for $\alpha \in\left[u_{n}, u_{n+1}\right)$ one can define a surjection of $u_{n}$ onto $\alpha$, and this together with Theorem 1.3 for subsets of $u_{n}$ allows proving that the complement of $B$ is $\Pi_{3}^{1}$. So $B$ itself is $\Sigma_{3}^{1}$. By Lemma 1.1, it has a $\Delta_{3}^{1}$ bound, say $\beta=|w|$ where $w$ is $\Delta_{3}^{1}$. Since $\beta$ bounds $B, A \cap \beta$ is non-empty. If $n=0$, it is easy to see that from $w$ one can define $\Delta_{3}^{1}$ codes for all ordinals smaller than $\beta$, and this includes in particular some $\alpha \in A$. If $n>0$ then using $w$ one can define a surjection $f: u_{n} \rightarrow \beta$, so that $f^{-1}(A \cap \beta)$ is $\Pi_{3}^{1}$, and for any $\Delta_{3}^{1}$ ordinal $\xi<u_{n}, f(\xi)$ is $\Delta_{3}^{1}$. By induction $f^{-1}(A \cap \beta)$ has a $\Delta_{3}^{1}$ element, and the image of this ordinal under $f$ is then a $\Delta_{3}^{1}$ element of $A$.

The following corollary is an application of the Kechris-Martin theorem. Together with Remark 1.5, it gives a characterization of the pointclass $\Pi_{3}^{1}$ in terms of definability over $T_{2}$, the Martin-Solovay tree for $\Pi_{2}^{1}$. (This is a tree on $\omega \times u_{\omega}$ that projects to a universal $\Pi_{2}^{1}$ set.)

Corollary 1.4 (Kechris-Martin [1]). For $x \in \mathbb{R}$, let $\alpha_{x}>u_{\omega}$ be least so that $L_{\alpha_{x}}\left(T_{2}, x\right)$ is admissible. Let $\psi$ be $\Sigma_{1}$. Then the set $\left\{x \mid L_{\alpha_{x}}\left(T_{2}, x\right) \models \psi\left(T_{2}, x\right)\right\}$ is $\Pi_{3}^{1}$.

REMARK 1.5. The converse of Corollary 1.4 is also true: every $\Pi_{3}^{1}$ set is of the form $\left\{x \mid L_{\alpha_{x}}\left(T_{2}, x\right) \models \psi\left(T_{2}, x\right)\right\}$ for a $\Sigma_{1}$ formula $\psi$. This is easily seen using the fact that $T_{2}$ projects to a universal $\Pi_{2}^{1}$ set.

In the following sections we will directly prove the corollary, using inner model theoretic methods. Using the same methods we will also prove Theorem 1.2 (which subsumes Lemma 1.1) and Theorem 1.3.

The proofs rely on approximations of the structures $L_{\alpha_{x}}\left(T_{2}, x\right)$ in inner models for one Woodin cardinal, and a lemma relating the approximations to the original structures. The approximations themselves are defined in Sections 4 and 5 . 
Given a model $N$, the approximation $T_{N}$ of $T_{2}$ in $N$ is simply (the transitive collapse of) the restriction of $T_{2}$ to ordinals with codes that belong to $N$. The approximation of $L_{\alpha_{x}}\left(T_{2}, x\right)$ is an initial segment of the least admissible structure $L_{\gamma_{x}^{N}}\left(T_{N}, x\right)$ in $N$ over $T_{N}$ and $x$.

Lemma 5.2 gives a certain correctness for approximations in sufficiently iterable inner models, showing that there is a $\Sigma_{1}$ elementary embedding of the approximations into the original structures. The key to its proof is Corollary 4.8 , that shows that an approximating structure in a sufficiently iterable model can be elementarily enlarged to absorb a code for any given ordinal below $u_{\omega}$.

Using the correctness of the approximating structures, $\Pi_{1}$ statements over $L_{\alpha_{x}}\left(T_{2}, x\right)$ can be reduced to statements about the existence of sufficiently iterable inner models whose approximating structures satisfy the given statements. The models are countable, hence coded by reals, and the iterability conditions used are $\Pi_{2}^{1}$. This allows connecting $\Pi_{1}$ statements over $L_{\alpha_{x}}\left(T_{2}, x\right)$ with $\Sigma_{3}^{1}$ conditions on reals, yielding a proof of Corollary 1.4. Similar computations using the approximations and the complexity of the iteration condition yield Theorems 1.2 and 1.3.

§2. Preliminaries. We assume that the reader is familiar with at least the basics of extenders and iteration trees. This section describes the results that we will need from the theory of fine structural inner models. These results mainly concern models satisfying weak conditions of iterability. The main result in the section is Lemma 2.2, which shows that a weak iterability condition of $\Pi_{2}^{1}$ complexity is enough to identify $H$ which can form the hereditarily countable sets of models with a Woodin cardinal that are fully iterable above their $\omega_{1}$. The weak notion of iterability that we use is due to Martin-Steel [2]. The lemma is implicit in the results of Section 6 of [2]. Using a stronger notion of weak iterability (but of the same projective complexity, $\Pi_{2}^{1}$ ), Woodin proved a stronger claim that produces fully iterable models, without having to assume their existence a priori, from the weakly iterable ones. But we will not go into this argument since it is not necessary for our purposes.

A model throughout the paper is a pair $\left\langle M, \mathcal{E}^{M}\right\rangle$, where $M$ satisfies ZFC and $\mathcal{E}^{M}$ is an $M$ class of extenders of $M$. Iterability for $M$ is always relative to $\mathcal{E}^{M}$, meaning that it applies only to trees using extenders from $\mathcal{E}^{M}$ and its images along the iteration. Large cardinal properties in $M$ are interpreted relative to $\mathcal{E}^{M}$. In particular, we say that $\delta$ is Woodin in $M$ just in case that this is witnessed by extenders in $\mathcal{E}^{M}$, or more precisely, just in case that for every function $f: \delta \rightarrow \delta$ in $M$, there is an extender $E \in \mathcal{E}^{M}$ which is $i_{E}^{M}(f)(\kappa)$ strong in $M$, where $\kappa=\operatorname{crit}(E)$ and $i_{E}^{M}$ is the ultrapower embedding of $M$ by $E$.

A model $M$ is almost fine structural if it is fine structural above its hereditarily countable sets. More precisely, $M$ is almost fine structural if it is of the form $M=L_{\nu}[\vec{E}](H)$, where $H$ consists of all hereditarily countable sets of $M$ (together with some predicate wellordering them in order type $\omega_{1}^{M}$ ), and $\vec{E}$ is a coherent sequence of extenders over $H$. The model is 1 -small if there is no $\delta$ so that $L[\vec{E}\lceil\delta](H) \models$ " $\delta$ is a Woodin cardinal" and $\vec{E}$ includes a sharp above $\delta$. We work with almost fine structural models below. Iteration trees on such models 
are allowed to use partial extenders on the sequence $\vec{E}$, leading to fine structural truncations along the tree. But the trees can only use extenders of $\vec{E}$, and since all these extenders are above $H$, with both extender index and critical point above $\omega_{1}^{M}$, this implies in particular that there are no truncations to countable initial segments of $M$. Another way to put this is that iterability in this paper (of any kind, including full) for almost fine structural models is always restricted to trees above the hereditarily countable sets of the model.

By an essentially countable model we mean a model $M$ of the form $L_{\nu}\left(V_{\delta}^{M}\right)$, where $\nu=\omega_{1}^{V}$, and $\delta$ is countable in $V$. The model is determined completely by $W=V_{\delta}^{M}$, and we say that $W$ extends to the essentially countable model $M$. Abusing notion, we sometimes say that a property holds of $W$ to mean that it holds of $M$.

A set $A$ of essentially countable models belongs to a pointclass $\Gamma$ if the set of structures $x$ on $\omega$ which are isomorphic to $V_{\delta}^{M}$ for $M=L_{\omega_{1}}\left(V_{\delta}^{M}\right) \in A$, belongs to $\Gamma$.

An essentially countable model $M$ is $\Pi_{2}^{1}$ iterable if for every countable, normal iteration tree $\mathcal{T}$ on $M$ and every $\alpha<\omega_{1}$, there is a maximal branch $b$ of $\mathcal{T}$ so that either: (a) $M_{b}=M_{b}^{\mathcal{T}}$ is wellfounded of ordinal height less than $\alpha$; or else (b) the wellfounded part of $M_{b}$ contains $\alpha$. (Option (a) can only occur if there are truncations along $b$, since otherwise $M_{b}$, if wellfounded, has ordinal height $\omega_{1}^{V}$.) $M_{b}=M_{b}^{\mathcal{T}}$ here is the direct limit of the models of $\mathcal{T}$ along $b$ (or a tail-end of $b$ in case of truncations). The set of $\Pi_{2}^{1}$ iterable essentially countable models is $\Pi_{2}^{1}$, hence the terminology name.

For $\xi<\operatorname{lh}(\mathcal{T})$, the branch $[0, \xi]_{\mathcal{T}}$ of $\mathcal{T}$ is called $\Sigma_{2}^{1}$ justified if there exists some countable $\alpha=\alpha_{\xi}$ so that $[0, \xi]_{\mathcal{T}}$ is the only cofinal branch of $\mathcal{T}\lceil\xi$ satisfying (a) or (b) of the previous paragraph. The tree $\mathcal{T}$ is $\Sigma_{2}^{1}$ justified if all branches $[0, \xi]_{\mathcal{T}}$ for $\xi<\operatorname{lh}(\mathcal{T})$ are $\Sigma_{2}^{1}$ justified. This is a $\Sigma_{2}^{1}$ condition on $\mathcal{T}$, hence the terminology name. If $\mathcal{T}$ is $\Sigma_{2}^{1}$ justified, and $M$ is $\Pi_{2}^{1}$ iterable, then for $\alpha \geq \sup \left\{\alpha_{\xi} \mid \xi<\operatorname{lh}(\mathcal{T})\right\}$, the maximal branch $b$ given by $\Pi_{2}^{1}$ iterability must be cofinal in $\mathcal{T}$.

Given a normal iteration tree $\mathcal{T}$ on $M$, we use $\Delta(\mathcal{T})$ to denote the lined-up part of $\mathcal{T}$, meaning the union $\bigcup_{\xi<\operatorname{lh}(\mathcal{T})} M_{\xi}^{\mathcal{T}} \| \operatorname{strength}\left(E_{\xi}^{\mathcal{T}}\right) . \delta(\mathcal{T})$ denotes On $\cap \Delta(\mathcal{T})$. Every strict initial segment of $\Delta(\mathcal{T})$ is an initial segment of $M_{\xi}^{\mathcal{T}}$ for all sufficiently large $\xi<\operatorname{lh}(\mathcal{T})$. (Indeed, $\Delta(\mathcal{T})$ is the largest model with this property.) This implies in particular that $\Delta(\mathcal{T})$ is an initial segment of $M_{b}$ for any cofinal branch $b$ of $\mathcal{T}$.

Claim 2.1. Let $M$ be essentially countable. Suppose that $M$ is $\Pi_{2}^{1}$ iterable, $\mathcal{T}$ is a normal iteration tree on $M$ of countable length, $\mathcal{T}$ is $\Sigma_{2}^{1}$ justified, and there is no $\Sigma_{2}^{1}$ justified cofinal branch through $\mathcal{T}$. Then $\mathcal{T}$ must have limit length, and $\delta(\mathcal{T})$ must be a Woodin cardinal in $L(\Delta(\mathcal{T})$ ).

Proof. The case of successor length is easily ruled out, using the $\Pi_{2}^{1}$ iterability of $M$ and $\Sigma_{2}^{1}$ justification of existing branches of $\mathcal{T}$ to ensure that the unique cofinal branch of $\mathcal{T}$ is $\Sigma_{2}^{1}$ justified. We work on the limit case.

If $\delta(\mathcal{T})$ fails to be Woodin in $L(\Delta(\mathcal{T}))$, then there is some countable $\gamma$ so that $\delta(\mathcal{T})$ fails to be Woodin already in $L_{\gamma}(\Delta(\mathcal{T}))$. By Martin-Steel [2], this implies that there can only be one cofinal branch $b$ of $\mathcal{T}$ so that $L_{\gamma}(\Delta(\mathcal{T})) \subseteq M_{b}$. Since 
$\Delta(\mathcal{T})$ is contained in all direct limits along cofinal branches of $\mathcal{T}$ it follows that there can only be one cofinal branch $b$ so that $\gamma \subseteq M_{b}$.

Using standard fine structural arguments it follows from this that there is at most one cofinal branch $b$ so that either $M_{b}$ is wellfounded of ordinal height $<\gamma$, or $\gamma$ is contained in the wellfounded part of $M_{b}$. (The former can only occur if there are truncations along $b$.) It remains to prove that a cofinal branch with one of these properties exists. $\Pi_{2}^{1}$ iterability of $M$ guarantees the existence of a maximal branch with one of these properties. Increasing $\gamma$ if needed we may assume it witnesses that all branches $[0, \xi]_{\mathcal{T}}$ for $\xi<\operatorname{lh}(\mathcal{T})$ are $\Sigma_{2}^{1}$ justified, and the resulting uniqueness implies that the maximal branch given by $\Pi_{2}^{1}$ iterability is in fact cofinal.

Lemma 2.2. Suppose that for every real $x$, there is a fully iterable inner model for the sharp of one Woodin cardinal over $x$. Let $M$ be essentially countable, almost fine structural, and 1 small. If $M$ is $\Pi_{2}^{1}$ iterable, then there is a fully iterable model $N$ (essentially countable, almost fine structural, and 1 small) with exactly the same reals.

Proof. If $M$ itself is fully iterable then there is nothing to prove. Suppose then that $M$ is not fully iterable.

Let $H$ consists of the hereditarily countable sets of $M$ (together with some predicate for a wellordering of $H$ that belongs to $M$ and has order type $\omega_{1}^{M}$ ). Recall that $M_{1}^{\sharp}(H)$ is the minimal fully iterable fine structural inner model for the sharp of one Woodin cardinal over $H$. It exists by assumptions of the lemma, as $H$ is countable in $V$. If all the reals in $M_{1}^{\sharp}(H)$ belong to $H$ (in other words to $M)$, then we can take $N$ to be $L_{\omega_{1}^{V}}\left(M_{1}^{\sharp}(H) \| \delta\right)$ where $\delta$ is the Woodin cardinal of $M_{1}^{\sharp}(H)$. The model is fully iterable, and has the same reals as $M$, namely the reals in $H$. (Recall that full iterability for an almost fine structural model involves only trees above the hereditarily countable sets of the model. This is the iterability that the claim states for $N$, and this iterability holds for $M_{1}^{\sharp}(H)$ in case its hereditarily countable sets are exactly the ones in $H$.)

Suppose then for contradiction that $M_{1}^{\sharp}(H)$ has reals outside $H$. Let $P$ be the first level of $M_{1}^{\sharp}(H)$ so that a real outside $H$ is definable over $P$. In particular then $P$ projects to $\omega$.

$M$ and $P$ are both fine structural above $H$, which has exactly their hereditarily countable sets. We may therefore form a fine structural comparison of the models above $H$. Let $\mathcal{T}$ on $M$ and $\mathcal{U}$ on $P$ be the trees of the comparison. $\mathcal{U}$ is formed using the iteration strategy for $P$. $\mathcal{T}$ is formed by picking unique $\Sigma_{2}^{1}$ justified branches when possible, ending the comparison at the first stage where there is no $\Sigma_{2}^{1}$ justified cofinal branch on the $M$ side.

Note that it is impossible for the end iterate of $P$ in the comparison to be a strict initial segment of the end iterate of $M$, since $P$ projects to $\omega$. Similarly it is impossible for the two end iterates to be equal if the $M$ side has a truncation. It is also impossible for the end iterate of $M$ to be a (possibly non-strict) initial segment of the end iterate of $P$ if there are no truncations on the $M$ side, since $M$ is not iterable, while all iterates of $P$ are. Thus the comparison must fail. As $P$ is fully iterable, there are only two possible reasons for this. One is that 
$\operatorname{lh}(\mathcal{T})<\omega_{1}$ and there is no $\Sigma_{2}^{1}$ justified cofinal branch through $\mathcal{T}$. The other is that $\ln (\mathcal{T})=\omega_{1}$ and there is no cofinal branch through $\mathcal{T}$.

Consider first the case that $\ln (\mathcal{T})<\omega_{1}$. $\mathcal{U}$ leads to a final model, call it $P^{*}$. $P^{*}$ extends $\Delta(\mathcal{T})$, and since $P$ projects to $\omega, P^{*}$ must project below $\delta(\mathcal{T})$. (If there are no truncations on the $P$ side of the comparison, $P^{*}$ projects to $\omega$. Otherwise it may project higher, but still below $\delta(\mathcal{T})$ as all extenders used in the comparison have critical points below $\delta(\mathcal{T})$.)

Since $M$ is $\Pi_{2}^{1}$ iterable, $\mathcal{T}$ is $\Sigma_{2}^{1}$ justified, and there is no $\Sigma_{2}^{1}$ justified cofinal branch through $\mathcal{T}$, by Claim $2.1, \delta(\mathcal{T})$ is Woodin in $L_{\omega_{1}^{V}}(\Delta(\mathcal{T}))$. It follows form this, the fact that $P^{*}$ extends $\Delta(\mathcal{T})$, and the fact that $P^{*}$ is 1 small, that $P^{*}$ cannot contain a sharp for $\Delta(\mathcal{T})$, and hence $P^{*}$ is an initial segment of $L_{\omega_{1}^{V}}(\Delta(\mathcal{T}))$.

But then since $P^{*}$ projects below $\delta(\mathcal{T})$, it follows that $\delta(\mathcal{T})$ is not a cardinal in $L_{\omega_{1}^{V}}(\Delta(\mathcal{T}))$, let alone a Woodin cardinal. This contradiction completes the proof in case $\ln (\mathcal{T})<\omega_{1}$.

The case that $\ln (\mathcal{T})=\omega_{1}$ is similar, but involves working in an extension $V[G]$ of $V$ by $\operatorname{col}\left(\omega, \omega_{1}\right) . M$ (or rather its natural lengthening to an essentially countable model in $V[G]$ ) continues to be $\Pi_{2}^{1}$ iterable in the extension. $\mathcal{T}$ is countable in the extension and has no $\Sigma_{2}^{1}$ justified cofinal branch. (If there were a $\Sigma_{2}^{1}$ justified branch in the extension, then its uniqueness, combined with the homogeneity of the collapse, would allow proving that the branch belongs to $V$.) This leads to a contradiction as in the case $\ln (\mathcal{T})<\omega_{1}$.

$\S 3$. Genericity iterations. The key tool we will use in proving Corollary 4.8 is a genericity iteration. Recall that genericity iterations are iterations of a model $M$ that allow absorbing an arbitrary real into a generic extension of the end iterate. The first genericity iteration was discovered by Woodin. Later on a second form of a genericity iteration was discovered by Neeman [3, 4]. The two iterations use different posets and give rise to iteration trees of different formats, a general comparison tree in the case of Woodin's genericity iterations, and a tree of length $\omega$ in the case of Neeman's iterations. We will use a combination of the two methods here, that will produce iteration trees of length $\omega$ to make the given real generic, while producing an additional real, generic for Woodin's extender algebra.

Given a cardinal $\delta$ in a model of ZFC, let $\mathbb{W}_{\delta}^{M}$ denote Woodin's extender algebra built using formulas and extenders of von-Neumann rank less than $\delta$ in the model $M$. Conditions in $\mathbb{W}_{\delta}$ are transfinite formulas $\varphi$ generated by negations, conjunctions of length $<\delta$, and disjunctions of length $<\delta$, from atomic formulas $\dot{x}(n)=m$ (where $n, m<\omega$ and $\dot{x}$ are symbols of the language). The ordering is a natural implication ordering given certain essential axioms that refer to and use extenders. We will not go into the exact nature of these axioms, but mention some of the resulting properties of $\mathbb{W}_{\delta}^{M}$. The first is the following:

Theorem 3.1 (Woodin). Suppose that $\delta$ is a Woodin cardinal in $M$. Then $\mathbb{W}_{\delta}^{M}$ is $\delta$-c.c. in $M$.

The definition of the extender algebra can also be made in case $\delta=$ On, resulting in a class poset. For any model $M$, the algebra $\mathbb{W}_{\delta}^{M}$ is equal to $\left(\mathbb{W}_{\text {On }}\right)^{V_{\delta}^{M}}$. 
In case $\delta$ is a Woodin cardinal of $M$, the fact that $\mathbb{W}_{\delta}^{M}$ is $\delta$-c.c. in $M$ implies that every maximal antichain of $\mathbb{W}_{\delta}^{M}$ in $M$ belongs to $V_{\delta}^{M}$. Thus, any filter $H$ that is generic for $\left(\mathbb{W}_{\mathrm{On}}\right)^{V_{\delta}^{M}}$ over $V_{\delta}^{M}$ is also generic for $\mathbb{W}_{\delta}^{M}$ over $M$.

Let $y$ be a real, which we view as a function from $\omega$ into $\omega$. Then $y$ generates a filter on $\mathbb{W}_{\delta}^{M}$. The filter, call it $H_{y}$, consists of all conditions $\varphi$ so that $y \models \varphi$. (A real $y$ satisfies an atomic formula $\dot{x}(n)=m$ iff $y(n)=m$. Satisfaction for formulas of greater complexity is defined in the natural way by transfinite induction.) We say that $y$ is generic for $\mathbb{W}_{\delta}^{M}$ over $M$ if $H_{y}$ is generic, and write $M[y]$ for $M\left[H_{y}\right]$. We say that $y$ is generic below a condition $\varphi$ if in addition $\varphi \in H_{y}$.

The real $y$ is easily defined from $H_{y}$, as $y(n)=m$ iff $\dot{x}(n)=m$ belongs to $H_{y}$. So $y$ belongs to the generic extension by $H_{y}$. We refer to $H_{y}$ as the filter generated by $y$, and to $y$ as the real determined by $H_{y}$.

We say that an extender of $M$ is below $\delta$ if it belongs to $V_{\delta}^{M}$.

Theorem 3.2 (Woodin). Suppose that $M$ is fully iterable, $\delta$ is a Woodin cardinal of $M$, and $V_{\delta}^{M}$ is countable in $V$. Then for every real $y$ (in $V$ ) there is a countable iteration tree $\mathcal{T}$ on $M$ using extenders below $\delta$, with a final model $M^{*}$ and final embedding $j: M \rightarrow M^{*}$, so that $y$ is generic for $\mathbb{W}_{j(\delta)}^{M^{*}}=j\left(\mathbb{W}_{\delta}^{M}\right)$ over $M^{*}$.

We refer the reader to Steel [5] for a definition of the extender algebra, and proofs of Theorems 3.1 and 3.2. Let us here only note that $\mathbb{W}_{\delta}$ is defined in such a way that extenders are obstructions to genericity. The proof of Theorem 3.2 is similar to a comparison argument, eliminating obstructions to the genericity of $x$ in much the same way that comparisons eliminate disagreements.

The genericity iteration in Neeman $[3,4]$ is of a different nature, constructing an iteration tree not through a transfinite process of eliminating obstructions, but through a continuous process of length $\omega$, derived from proofs of determinacy.

Theorem 3.3 (Neeman $[3,4]$ ). Suppose that $\delta$ is a Woodin cardinal in $M$, and $V_{\delta+1}^{M}$ is countable in $V$. Then there is a continuous function which associates to every real $z$, a length $\omega$ iteration tree $\mathcal{T}=\mathcal{T}(z)$ using extenders below $\delta$, with a distinguished branch called the even branch, so that:

1. The even branch of $\mathcal{T}$ leads to an illfounded model.

2. For every odd branch $b$ (meaning a branch $b$ other than the even branch), there a generic $g$ for $\operatorname{col}\left(\omega, j_{b}(\delta)\right)$ over $M_{b}$ so that $z \in M_{b}[g]$.

Moreover, $\delta$ is fixed by all cofinal branches through the tree, the extenders used have strengths cofinal in $\delta$, and given any $\kappa<\delta$, one can arrange that all critical points used in $\mathcal{T}$ are greater than $\kappa$. A code for such a continuous function $z \mapsto \mathcal{T}(z)$ exists in any extension of $M$ by $\operatorname{col}(\omega, \delta)$.

The map $j_{b}$ in the theorem is the direct limit embedding of models of $\mathcal{T}$ along $b$, and $M_{b}$ is the direct limit model. If $M$ is iterable, then $\mathcal{T}$ has a branch $b$ so that $M_{b}$ is wellfounded. Since the even branch of $\mathcal{T}$ leads to an illfounded model, $b$ must be odd, and hence by condition (2), $z$ belongs to a generic extension of $M_{b}$. 
For the arguments in this paper we need a certain combination of the two methods for making reals generic. This combination is given by the next corollary. It uses trees of length $\omega$, as in Theorem 3.3, to make a given real $x$ and an additional real $y$ generic for the collapse, while at the same time making $y$ generic for the extender algebra below a given condition $\varphi$.

Corollary 3.4. Suppose that $\delta$ is a Woodin cardinal in $M$, and $V_{\delta+1}^{M}$ is countable in $V$. Let $\mathbb{W}=\mathbb{W}_{\delta}^{M}$. Let $\varphi$ be a condition in $\mathbb{W}$. Then there is a continuous function which associates to every real $x$, a length $\omega$ iteration tree $\mathcal{T}=\mathcal{T}(x)$ using extenders below $\delta$ and a real $y=y(x)$, so that:

1. The even branch of $\mathcal{T}$ leads to an illfounded model.

2. For every branch $b$ of $\mathcal{T}, j_{b}(\varphi)=\varphi$ and $y$ is generic for $j_{b}(\mathbb{W})$ over $M_{b}$, below the condition $\varphi=j_{b}(\varphi)$.

3. For every odd branch $b$, there is a generic $g$ for $\operatorname{col}\left(\omega, j_{b}(\delta)\right)$ over $M_{b}$ so that both $x$ and $y$ belong to $M_{b}[g]$.

$A$ code for such a continuous function $x \mapsto \mathcal{T}(x), y(x)$ exists in any extension of $M$ by $\operatorname{col}(\omega, \delta)$.

Proof. Let $\kappa<\delta$ be large enough that $\varphi \in V_{\kappa}^{M}$. Such $\kappa$ exist since $\mathbb{W} \subseteq V_{\delta}^{M}$.

Let $f$ denote the continuous map given by Theorem 3.3, associating to each real $z$ an iteration tree $\mathcal{T}=f(z)$, with all its critical points greater than $\kappa$, fixing $\delta$, and using extenders of strengths cofinal in $\delta$, to make $z$ generic over direct limits along the odd branches. We think of $z$ as coding a pair $\langle x, y\rangle$ of reals, and will refer to $f(z)$ as $f(x, y)$.

Given $x$, construct $y, \vec{\varphi}=\left\langle\varphi_{i} \mid i<\omega\right\rangle$, and $\mathcal{T}$ simultaneously, so that:

1. $\mathcal{T}=f(x, y)$.

2. $\vec{\varphi}$ is a descending sequence in $\left(\mathbb{W}_{\text {On }}\right)^{\Delta(\mathcal{T})}, \varphi_{0}=\varphi$, and $\vec{\varphi}$ meets the downward closure of every maximal antichains of $\left(\mathbb{W}_{\text {On }}\right)^{\Delta(\mathcal{T})}$ in $\Delta(\mathcal{T})$.

3. $y(n)=m$ iff for some $i, \varphi_{i}$ is stronger than $\dot{x}(n)=m$.

These objects can be constructed continuously in $x$, since $f$ is continuous, and since $\Delta(\mathcal{T})$ is determined continuously from $\mathcal{T}$. (More precisely, the sequence of restrictions $\Delta(\mathcal{T})\left\|\operatorname{strength}\left(E_{i}^{\mathcal{T}}\right)=M_{i}^{\mathcal{T}}\right\| \operatorname{strength}\left(E_{i}^{\mathcal{T}}\right)$ is determined continuously from $x$, and converges to $\Delta(\mathcal{T})$.)

By conditions (2) and (3), the upward closure $H$ of $\left\{\varphi_{i} \mid i<\omega\right\}$ is generic for $\left(\mathbb{W}_{\text {On }}\right)^{\Delta(\mathcal{T})}$ over $\Delta(\mathcal{T})$, and the real it determines is $y$. Since $\delta=j_{b}(\delta)$ is a Woodin cardinal in $M_{b}$ for every cofinal branch $b$ of $\mathcal{T}$, and $V_{\delta}^{M_{b}}=\Delta(\mathcal{T})$, this implies that $H$, and hence $y$, is generic for $j_{b}(\mathbb{W})=\mathbb{W}_{\delta}^{M_{b}}$ over $M_{b}$, as required for condition (2) of the corollary. Conditions (1) and (3) are immediate from the use of Theorem 3.3.

§4. The main lemma. We work now in a universe satisfying ZF $+D C+$ "for every real $x$ there is a fully iterable inner model for the sharp of one Woodin cardinal over $x$ ". By a result of Woodin, the inner models assumption is a consequence of, and indeed equivalent to, $\boldsymbol{\Delta}_{2}^{1}$ determinacy.

Let $M$ be essentially countable. Note that since the ordinal height of $M$ is $\omega_{1}^{V}$, $M$ is correct about being a sharp. Precisely, if $z$ is a real in $M$ and $M \models z=r^{\sharp}$, then $z$ is indeed equal to $r^{\sharp}$. It follows from this that being a code for an ordinal 
less than $u_{\omega}$ reflects from $M$ to $V$. The correctness of $M$ about being a sharp also implies correctness about the restriction of $T_{2}$ to ordinal codes in $M$. The following fact phrases this precisely. Its proof relies on the definition of $T_{2}$. For our purposes it can be taken as a property of the tree. It is the only property of the tree that we will use in proving Theorem 1.4.

FACT 4.1. There is a formula $\psi$ so that for every essentially countable $M$ with the reals of $M$ closed under sharps, every sequence $\left\langle w_{0}, \ldots, w_{i-1}\right\rangle \in M$ of codes for ordinals below $u_{\omega}$, and every sequence $\left\langle n_{0}, \ldots, n_{i-1}\right\rangle$ of natural numbers, $\left\langle n_{0},\left|w_{0}\right|, \ldots, n_{i-1},\left|w_{i-1}\right|\right\rangle$ is a node in $T_{2}$ iff $M \models \psi\left(\left\langle n_{0}, w_{0}, \ldots, n_{i-1}, w_{i-1}\right\rangle\right)$.

Define $Z_{M}$ to be the set of ordinals below $u_{\omega}$ with codes in $M$. Let $X_{M}$ be the transitive collapse of $Z_{M}$, and let $c=c_{M}: Z_{M} \rightarrow X_{M}$ be the transitive collapse embedding. Since $M$ is correct about codes for ordinals below $u_{\omega}$, the function $w \mapsto c(|w|)$, on $w \in M$ which are codes for ordinals below $u_{\omega}$, belongs to $M$. Let $T_{M}$ be the image of $T_{2} \uparrow\left(\omega \times Z_{M}\right)$ under $c$. $T_{M}$ is a tree on $\omega \times X_{M}$, and by Fact 4.1 it belongs to $M$.

For each real $x \in M$ let $\gamma_{x}=\gamma_{x}^{M}$ be the least ordinal above $\sup \left(X_{M}\right)$ so that $L_{\gamma_{x}}\left(T_{M}, x\right)$ is admissible. Let $\gamma_{M}=\sup \left\{\gamma_{x} \mid x \in M\right\}$. Let $\mathfrak{A}_{M}$ be the structure $L_{\gamma_{M}}\left(\mathbb{R}^{M}, T_{M}\right)$. Since $\mathfrak{A}_{M}$ has all the reals of $M$, it can identify which $w \in M$ are codes, and compute the map $w \mapsto c(|w|)$.

REMARK 4.2. The important properties of $\mathfrak{A}_{M}$ that we will use later on are:

1. $\mathfrak{A}_{M}$ belongs to $M$, and depends only on the reals of $M$.

2. If $\sigma: M \rightarrow M^{*}$ is elementary, then the restriction of $\sigma$ to $\mathfrak{A}_{M}$ is an elementary embedding of $\mathfrak{A}_{M}$ into $\mathfrak{A}_{M^{*}}$.

3. If $\sigma: \mathfrak{A}_{M} \rightarrow \mathfrak{A}_{M^{*}}$ is elementary, then for every real $x \in M$, the restriction $\pi$ of $\sigma$ to $L_{\gamma_{x}^{M}}\left(T_{M}, x\right)$ is elementary from $L_{\gamma_{x}^{M}}\left(T_{M}, x\right)$ into $L_{\gamma_{x}^{M^{*}}}\left(T_{M^{*}}, x\right)$, with the property that for each code $w \in M, \pi\left(c_{M}(|w|)\right)=c_{M^{*}}(|w|)$.

4. If $\sigma: \mathfrak{A}_{M} \rightarrow \mathfrak{A}_{M^{*}}$ is elementary, $a \in \mathfrak{A}_{M}$, and there is a real $y \in M^{*}$ so that $L_{\gamma_{M^{*}}}\left(T_{M^{*}}, \sigma(a), y\right) \models \theta\left(T_{M^{*}}, \sigma(a), y\right)$, then there is a real $y \in M$ so that $L_{\gamma_{M}}\left(T_{M}, a, y\right) \models \theta\left(T_{M}, a, y\right)$.

These properties are immediate from the definition. The fact that $\pi\left(c_{M}(|w|)\right)=$ $c_{M^{*}}(|w|)$ in condition (3) relies on the observation that $\mathfrak{A}_{M}$ can compute the map $w \mapsto c(|w|)$.

For a real $v$ let $v^{+}$denote the first admissible above $\omega_{1}^{V}$ in $L(v)$. Let $v_{\alpha}^{+}$denote the first admissible in $L(v)$ above $\alpha$.

Call $y$ a putative sharp for $r$ if $y$ codes a complete, consistent theory of $\omega$ indiscernible ordinals, with the sentence $V=L(\dot{r})$, the sentences $\dot{r}(n)=m$ for all $m, n<\omega$ so that $r(n)=m$, and the sentences $\dot{r}(n) \neq m$ for $m, n<\omega$ so that $r(n) \neq m$. We refer to $r$ as $r_{y}$. Given an ordinal $\rho$, we use $y[\rho]$ to denote the theory of $\rho$ (increasing) indiscernibles, satisfying the sentences of $y$. Precisely this means that the restriction of $y[\rho]$ to any finite set of indiscernibles is equal to the restriction of $y$ to any set of indiscernibles of the same size. Then $y[\rho]$ is complete and consistent. Since it includes the sentence $V=L(\dot{r})$, it has a minimal model up to isomorphism. This is the model where every element is definable from finitely many indiscernibles. We use $S(y, \rho)$ to denote this model. The putative sharp $y$ is $\rho$ iterable if $S(y, \rho)$ is wellfounded, and $y$ is a true sharp 
if for every $\rho$ (equivalently every $\left.\rho<\omega_{1}\right), S(y, \rho)$ is wellfounded. In this case $y$ is equal to $r^{\sharp}$.

The set of putative sharps is Borel. The set of true sharps is $\Pi_{2}^{1}$. We will work with putative sharps that are partially iterable. Let $\alpha<\omega_{1}$. A putative sharp $y$ reaches $\alpha$ if there exists some $\rho$ so that $\alpha+1$ is contained in the wellfounded part of $S(y, \rho)$. Note that the least $\rho$ witnessing this is smaller than or equal to $\alpha+1$. It follows that the set of putative sharps which reach $\alpha$ is $\Sigma_{1}^{1}$ in a code for $\alpha$.

For a putative sharp $y$ that reaches $\alpha$, let $t(y, \alpha)$ be the order type of the ordinals in $S(y, \rho)$, where $\rho \leq \alpha+1$ is the least witness that $y$ reaches $\alpha$. From $y$ and any linear order on $\omega$ of order type $\rho$, one can recursively obtain a linear order on $\omega$ of order type $t(y, \alpha)$. It follows that there is a $\Sigma_{1}^{1}$ function $U$ so that: (a) the domain of $U$ contains the set of pairs $\langle y, a\rangle$ where $a$ is a wellorder on $\omega$, $y$ is a putative sharp, and $y$ reaches the order type of $a$, call it $\alpha$; and (b) $U(y, a)$ is a linear order on $\omega$ of order type $t(y, \alpha)$. We will use this function in the proof of the next lemma. For now, let us just note that if $t(y, \alpha)=$ o.t. $(U(y, a))$ is wellfounded, then it is greater than $r_{\alpha}^{+}$(where $r=r_{y}$ ), since $S(y, \rho)$ is a model of ZFC and hence certainly admissible.

Lemma 4.3. Let $M$ be essentially countable, almost fine structural, and fully iterable. Suppose that $\delta=\delta_{M}$ is a Woodin cardinal of $M$, and $V_{\delta+1}^{M}$ is countable in $V$. Let $r$ be a given real. Then there is an essentially countable, almost fine structural, $\Pi_{2}^{1}$ iterable model $N$, with a Woodin cardinal $\delta_{N}$, so that:

1. There is an elementary embedding from $\mathfrak{A}_{M}$ into $\mathfrak{A}_{N}$.

2. There is a real $v \in N$ with $v^{+}>r^{+}$.

Proof. Call a model $N$ pre-nice if it is essentially countable, almost fine structural, and has a Woodin cardinal $\delta_{N}$.

Suppose the lemma fails. Let $M^{*}$ be the result of iterating $M$ to make the real $r^{\sharp}$ generic for Woodin's extender algebra, let $j: M \rightarrow M^{*}$ be the iteration embedding, and let $\delta^{*}=j(\delta)$. Let $\mathbb{W}^{*}=j\left(\mathbb{W}_{\delta}^{M}\right)=\mathbb{W}_{\delta^{*}}^{M^{*}}$. Since $M$ and $M^{*}$ have the same reals, $\mathfrak{A}_{M^{*}}=\mathfrak{A}_{M}$. The following statement $(\dagger)$ is then true of $r^{\sharp}$ in $M^{*}\left[r^{\sharp}\right]: r^{\sharp}$ is a true sharp and for every pre-nice $N$ added by $\operatorname{col}\left(\omega, \delta^{*}\right)$, either

1. there is no elementary embedding from $\mathfrak{A}_{M^{*}}$ into $\mathfrak{A}_{N}$, or

2. $N$ is not $\Pi_{2}^{1}$ iterable, or

3. for every $v \in N$, there is an ordinal $\alpha$ so that $v_{\alpha}^{+} \leq r_{\alpha}^{+}$.

This statement follows immediately from our assumption for contradiction that the lemma fails, noting that $M^{*}\left[r^{\sharp}\right]$ has ordinal height $\omega_{1}^{V}$, hence $\Pi_{2}^{1}$ iterability reflects from $M^{*}\left[r^{\sharp}\right]$ to $V$, and if $v_{\alpha}^{+}>r_{\alpha}^{+}$for all $\alpha \in M^{*}\left[r^{\sharp}\right]$, then $v^{+}>r^{+}$.

Let $\varphi^{*}$ be a condition in $\mathbb{W}^{*}$ that forces $(\dagger)$ over $M^{*}$. By reflection in $M^{*}$, there is some initial segment $P^{*}$ of $M^{*}$ (large enough that $\delta^{*}, \mathbb{W}^{*} \in P^{*}$ ) so that $\varphi^{*}$ forces $(\dagger)$ over $P^{*}$. Let $P$ be the transitive collapse of the Skolem hull of $\delta^{*}$ and $\varphi^{*}$ in $P^{*}$. Let $\varphi$ be the collapsed image of $\varphi^{*}$. Then $P$ is a countable transitive model inside $M^{*}$, and therefore belongs to $M$. The condition $\varphi$ forces $(\dagger)$ over $P$.

$P$ is fully iterable, since it embeds into an initial segment $P^{*}$ of $M^{*}$. Again since $P$ embeds into $P^{*}, \mathfrak{A}_{P}$ embeds elementarily into $\mathfrak{A}_{P^{*}}$, which in turn is equal 
to $\mathfrak{A}_{M^{*}}=\mathfrak{A}_{M}$ as $P^{*}$ and $M^{*}$ have the same reals. So $\mathfrak{A}_{P}$ embeds elementarily into $\mathfrak{A}_{M}$.

Let $\mathcal{T}(x)$ and $y(x)$ be given by Corollary 3.4, iterating $P$ below $\delta_{p}$ to make a real $x$ generic for the collapse of $\delta_{P}$, and getting $y(x)$ to be generic for the extender algebra below the condition $\varphi$.

Let $b$ be an odd branch of $\mathcal{T}(x)$, and let $g$ be generic for $\operatorname{col}\left(\omega, j_{b}\left(\delta_{P}\right)\right)$ so that $x$ and $y(x)$ belong to $P_{b}[g]$.

We will later use these assignments with $x$ equal to a real that codes $V_{\delta}^{M}$ and an elementary embedding of $\mathfrak{A}_{P}=\mathfrak{A}_{P_{b}}$ into $\mathfrak{A}_{M}$. For now let us just assume that $x$ codes some structure $W$ which extends to an essentially countable model in $P_{b}[g]$, and an elementary embedding of $\mathfrak{A}_{P}$ into $\mathfrak{A}_{W}$. Under these assumptions, it follows from the fact that $y(x)$ is generic for the extender algebra of $P_{b}$ below the condition $\varphi=j_{b}(\varphi)$ which forces $(\dagger)$ over $P$, that:

(i) In $P_{b}[g], y(x)$ is the sharp of a real $r(x)=r_{y(x)}$.

(ii) In $P_{b}[g]$, either $W$ is not $\Pi_{2}^{1}$ iterable, or else for every $v \in W$ there is an ordinal $\alpha$ so that $v_{\alpha}^{+} \leq r(x)_{\alpha}^{+}$.

The reference to the iterability of $W$ is an abuse of notation. Precisely we mean that the essentially countable model that $W$ extends to in $P_{b}[g]$ is not $\Pi_{2}^{1}$ iterable in $P_{b}[g]$.

Condition (i) does not imply that $y(x)$ is a true sharp, since $P_{b}$ need not be wellfounded, and in any case even if it is wellfounded, its height is smaller than $\omega_{1}^{V}$. However the condition implies some amount of iterability for $y(x)$, as follows. Let $b(x)$ be a wellfounded branch through $\mathcal{T}(x)$. Such a branch exists since $P$ is iterable, and by Corollary 3.4, the branch must be odd. Then, since condition (i) holds with $b=b(x)$, and since $P_{b(x)}$ is wellfounded, we have:

(iii) For every $\rho \in P_{b(x)}, y(x)$ is $\rho$ iterable.

Using the observations preceding Lemma 4.3 it follows that:

(iv) For every $\hat{\alpha} \in P_{b(x)}$, and every linear order $a$ on $\omega$ of order type $\hat{\alpha}, y(x)$ reaches $\hat{\alpha}$, and $t(y, \hat{\alpha})=$ o.t. $(U(y, a))$ is wellfounded and greater than $r(x)_{\hat{\alpha}}^{+}$.

Claim 4.4. Suppose $\hat{\alpha}$ is an ordinal so that $W$ is $\Pi_{2}^{1}$ iterable in $L_{\hat{\alpha}}(W)$, and there exists $v \in W$ so that $v_{\alpha}^{+}>r(x)_{\alpha}^{+}$for all $\alpha<\hat{\alpha}$. Then $\hat{\alpha}$ belongs to $P_{b(x)}$.

Proof. If $\hat{\alpha}$ does not belong to $P_{b(x)}$, then the ordinal height of $P_{b(x)}$ is at most $\hat{\alpha}$. By the assumptions of the claim it follows that, in $P_{b(x)}[g], W$ is $\Pi_{2}^{1}$ iterable, and $v_{\alpha}^{+}>r(x)_{\alpha}^{+}$for all $\alpha$. (The latter assertion is immediate from the assumptions of the claim and the fact that $P_{b(x)}$ has ordinal height at most $\hat{\alpha}$. The former uses also the fact that failure of $\Pi_{2}^{1}$ iterability is a $\Sigma_{2}^{1}$ statement and therefore reflects from $P_{b(x)}[g]$ to any wellfounded model of the same or greater ordinal height.)

But this contradicts condition (ii) for $b=b(x)$.

Claim 4.5. Suppose that the structure $W$ coded by $x$ is equal to $V_{\delta}^{M}$. Let $v=P^{\sharp}$. Then $v \in W$, and there exists an ordinal $\alpha$ so that $v_{\alpha}^{+} \leq r(x)_{\alpha}^{+}$and $W$ is $\Pi_{2}^{1}$ iterable in $L_{\alpha}(W)$. 
Proof. Note to begin with that $P$ belongs to $M$ by definition, and since $M$ is closed under sharps for reals, so does $v=P^{\sharp}$. Since $W=V_{\delta}^{M}$ and $M$ have the same reals, $v \in W$.

Let $b=b(x) . \quad M$ is fully iterable, hence certainly $\Pi_{2}^{1}$ iterable. Since $P_{b}$ is wellfounded, $\Pi_{2}^{1}$ iterability reflects to $P_{b}[g]$. So $W$ is $\Pi_{2}^{1}$ iterable in $P_{b}[g]$. Similarly $W$ is $\Pi_{2}^{1}$ iterable in $L_{\alpha}(W)$ for all ordinals $\alpha$.

Since $W$ is $\Pi_{2}^{1}$ iterable in $P_{b}[g]$, it follows by condition (ii) that in $P_{b}[g]$, there is an ordinal $\alpha$ so that $v_{\alpha}^{+} \leq r(x)_{\alpha}^{+}$. Since $P_{b}$ is wellfounded, $\alpha$ is an ordinal of $V$, and $v_{\alpha}^{+} \leq r(x)_{\alpha}^{+}$holds in $V$.

Let $\hat{\alpha}$ be the smallest ordinal witnessing Claim 4.5. Let $a$ be a linear order on $\omega$, of order type $\hat{\alpha}$, and let $p$ be a real coding $V_{\delta_{P}+1}^{P}$. Let $Z$ be the set of tuples $\langle x, \mathcal{T}, y, b, g\rangle$ so that:

- $\mathcal{T}=\mathcal{T}(x) ; y=y(x) ; b$ is an odd branch of $\mathcal{T} ; g$ is generic for $\operatorname{col}\left(\omega, j_{b}\left(\delta_{P}\right)\right)$ over $P_{b}$, with $x, y \in P_{b}[g] ; x$ codes a structure $W$ which extends to an essentially countable model in $P_{b}[g]$, and an elementary embedding of $\mathfrak{A}_{P}$ into $\mathfrak{A}_{W}$.

- $W$ is $\Pi_{2}^{1}$ iterable in $L_{\hat{\alpha}}(W)$.

- For every $\alpha<\hat{\alpha}, r(x)_{\alpha}^{+} \leq \hat{\alpha}$.

- There exists $v \in W$ so that for every $\alpha<\hat{\alpha}, v_{\alpha}^{+}>r(x)_{\alpha}^{+}$.

(The first item in the definition of $Z$ simply summarizes the assumptions we have been working under so far. The remaining items are new.)

It is clear that $Z$ is $\Sigma_{1}^{1}$ in $a$ and $p$. Let us just note that the continuous map of Corollary 3.4 can be recovered from $p$ since $p$ allows defining a generic for $\operatorname{col}\left(\omega, \delta_{P}\right)$ over $P$, that the references to $P_{b}$ only involve its restriction to $j_{b}\left(\delta_{P}\right)+1$, which can be determined from $p$, and that the final clauses, on $\Pi_{2}^{1}$ iterability of $W, r(x)_{\alpha}^{+}$, and $v$, only involve quantification over ordinals up to $\hat{\alpha}=$ o.t. $(a)$. (It is possible that for some $\alpha<\hat{\alpha}, v_{\alpha}^{+}>\hat{\alpha}$. But $r(x)_{\alpha}^{+}$is at most $\hat{\alpha}$, and for the final clause in the definition of $Z$ one need only express the fact that no ordinal in the interval $\left(\alpha, r(x)_{\alpha}^{+}\right]$is admissible relative to $v$.)

Claim 4.6. For every tuple $\langle x, \mathcal{T}, y, b, g\rangle$ in $Z, y$ reaches $\hat{\alpha}$, and $t(y, \hat{\alpha})=$ o.t. $(U(y, a))$ is wellfounded and greater than $r(x)_{\hat{\alpha}}^{+}$.

Proof. Fix $\langle x, \mathcal{T}, y, b, g\rangle$ in $Z$. By definition of $Z, W$ is $\Pi_{2}^{1}$ iterable in $L_{\hat{\alpha}}(W)$, and there exists $v \in W$ so that $v_{\alpha}^{+}>r(x)_{\alpha}^{+}$for all $\alpha<\hat{\alpha}$. It follows by Claim 4.4 that $\hat{\alpha}$ belongs to $P_{b(x)}$. By condition (iv) it follows that $y(x)$ reaches $\hat{\alpha}$, and $t(y, \hat{\alpha})=$ o.t. $(U(y, a))$ is wellfounded and greater than $r(x)_{\hat{\alpha}}^{+}$.

Let $\gamma=\sup \{$ o.t. $(U(y, a)) \mid\langle x, \mathcal{T}, y, b, g\rangle \in Z\}$. The order types are wellfounded by the previous claim. The function $U$ is $\Sigma_{1}^{1}$, and $Z$ is $\Sigma_{1}^{1}$ in $a$ and $p$. It follows using $\Sigma_{1}^{1}$ boundedness that $\gamma$ is smaller than the first admissible of $L(P)$ above $\hat{\alpha}$. (The use of $\Sigma_{1}^{1}$ boundedness requires appropriate choices of the codes $a$ and $p$ for $\hat{\alpha}$ and $V_{\delta_{P}+1}^{P}$. Any codes resulting from enumerations that are generic over $L(P)$ will do.) This, together with the final part of the previous claim, implies that for every tuple $\langle x, \mathcal{T}, y, b, g\rangle$ in $Z, r(x)_{\hat{\alpha}}^{+}$is smaller than the first admissible of $L(P)$ above $\hat{\alpha}$.

But consider the case that $x$ is taken to code the structure $W$ equal to $V_{\delta}^{M}$, and an embedding of $\mathfrak{A}_{P}$ into $\mathfrak{A}_{M}$. By Claim 4.5, and since $\hat{\alpha}$ was taken to 
be a witness for the claim, $W$ is $\Pi_{2}^{1}$ iterable in $L_{\hat{\alpha}}(W)$, and $v_{\hat{\alpha}}^{+} \leq r(x)_{\hat{\alpha}}^{+}$where $v=P^{\sharp} \in W$. Moreover, since $\hat{\alpha}$ was taken to be the least witness for the claim, $v_{\alpha}^{+}>r(x)_{\alpha}^{+}$for all $\alpha<\hat{\alpha}$. The minimality of $\hat{\alpha}$ also implies that $\hat{\alpha}$ itself is admissible or a limit of admissible ordinals relative to $r(x)$. Combining all these facts it follows that the tuple $\langle x, \mathcal{T}, y, b, g\rangle$ (for $x$ so that $W=V_{M}^{\delta}$ ) belongs to $Z$, that the element $v \in W$ witnessing the final clause in the definition of $Z$ is $v=P^{\sharp}$, and that $v_{\hat{\alpha}}^{+} \leq r(x)_{\hat{\alpha}}^{+}$. Applying the boundedness proved in the previous paragraph we get as a consequence that $v_{\hat{\alpha}}^{+}$is smaller than the first admissible of $L(P)$ above $\hat{\alpha}$. In other words, as $v=P^{\sharp}$, the first admissible of $L\left(P^{\sharp}\right)$ above $\hat{\alpha}$ is smaller than the first admissible of $L(P)$ above $\hat{\alpha}$. This of course is a contradiction. It completes the proof of Lemma 4.3.

LEMmA 4.7. Let $M$ be essentially countable, almost fine structural, and fully iterable. Suppose that $\delta=\delta_{M}$ is a Woodin cardinal of $M$, and $V_{\delta+1}^{M}$ is countable in $V$. Let $\alpha$ be a countable ordinal of $V$. Then there is an essentially countable, almost fine structural, $\Pi_{2}^{1}$ iterable model $N$, with a Woodin cardinal $\delta_{N}$, so that:

1. There is an elementary embedding from $\mathfrak{A}_{M}$ into $\mathfrak{A}_{N}$.

2. $\alpha$ belongs to $N$ and is countable in $N$.

Proof. This is similar to Lemma 4.3, but substantially simpler, since we are dealing here with countable ordinals, while Lemma 4.3 involved ordinals $r^{+}$and $v^{+}$above $\omega_{1}$.

Fix $\alpha$, and suppose the lemma fails. Let $M^{*}$ be obtained from $M$ by iterating ultrapowers by the first measure $\alpha+1$ times, so that $M^{*}$ is still essentially countable with a Woodin cardinal that is countable in $V$, and $\alpha$ is smaller than the first measurable cardinal of $M^{*}$. Since the lemma is assumed to fail, $M^{*}$ satisfies the following statement $(\dagger)(\alpha)$ : it is forced in $\operatorname{col}\left(\omega, \delta_{M^{*}}\right)$ that for every pre-nice $N$ in the extension, either $N$ is not $\Pi_{2}^{1}$ iterable, or there is no elementary embedding of $\mathfrak{A}_{M^{*}}$ into $\mathfrak{A}_{N}$, or $\alpha$ is not a countable ordinal in $N$. (As in the proof of Lemma 4.3, $N$ is pre-nice iff it is essentially countable, almost fine structural, with a Woodin cardinal $\delta_{N}$ which is countable in $V$.)

By reflection there is an initial segment $P^{*}$ of $M^{*}$ which satisfies $(\dagger)(\alpha)$. Let $X$ be the Skolem hull of $\delta_{M^{*}}$ and $\alpha$ in $P^{*}$, let $P$ be the transitive collapse of $X$, and let $\bar{\alpha}$ and $\delta_{P}$ be the images of $\alpha$ and $\delta_{M^{*}}$ under the collapse embedding. Note that $\bar{\alpha}$ is then countable in $M^{*}$, and hence also countable in $M$.

Since $P$ embeds into $P^{*}, \mathfrak{A}_{P}$ embeds elementarily into $\mathfrak{A}_{P^{*}}=\mathfrak{A}_{M^{*}}=\mathfrak{A}_{M}$. By elementarity of the collapse embedding, $P$ satisfies $(\dagger)(\bar{\alpha})$.

$P$ is fully iterable since it embeds into an initial segment of an iterate of $M$. Iterate $P$ to make a real coding $M$ and an elementary embedding of $\mathfrak{A}_{P}$ into $\mathfrak{A}_{M}$ generic. Let $Q$ be the end iterate, and let $j: P \rightarrow Q$ be the iteration embedding. Since $\bar{\alpha}$ is below the smallest measurable cardinal of $P$, it is not moved by $j$. By elementarity of $j$ then, $Q$ satisfies $(\dagger)(\bar{\alpha})$. In other words, it is forced in $\operatorname{col}\left(\omega, \delta_{Q}\right)$ over $Q$ that for every pre-nice $N$ that belongs to the extension, either $N$ is not $\Pi_{2}^{1}$ iterable, or there is no elementary embedding of $\mathfrak{A}_{Q}$ into $\mathfrak{A}_{N}$, or $\bar{\alpha}$ is not a countable ordinal in $N$. But $M$ is a counterexample to this: $M$ and an elementary embedding of $\mathfrak{A}_{Q}=\mathfrak{A}_{P}$ into $\mathfrak{A}_{M}$ belong to an extension of $Q$ by $\operatorname{col}\left(\omega, \delta_{Q}\right), M$ is fully iterable hence certainly $\Pi_{2}^{1}$ iterable in the extension, and 
$\bar{\alpha}$ is a countable ordinal in $M$. This contradiction completes the proof of the lemma.

Corollary 4.8. (Assuming that for every real $x$, there is a fully iterable model for the sharp of one Woodin cardinal over $x$.) Let $N$ be essentially countable, almost fine structural, $\Pi_{2}^{1}$ iterable model with a Woodin cardinal. Let $\alpha<u_{\omega}$. Then there is an essentially countable, almost fine structural, $\Pi_{2}^{1}$ iterable model $N^{*}$ with a Woodin cardinal, so that:

1. There is a code $w$ for $\alpha$ that belongs to $N^{*}$.

2. $\mathfrak{A}_{N}$ embeds elementarily into $\mathfrak{A}_{N^{*}}$.

Proof. The case $\alpha<\omega$ is trivial, so suppose $\alpha \geq \omega$. Let $n$ be least so that $\alpha$ belongs to $\left[u_{n}, u_{n+1}\right)$. We prove the corollary by induction on $n$.

The assumption in the corollary that over every real there is a fully iterable model for the sharp of a Woodin cardinal, allows us to use Lemma 2.2. By the lemma, there is a fully iterable, essentially countable and almost fine structural $M$ with a Woodin cardinal, so that $N$ and $M$ have the same reals. In particular then $\mathfrak{A}_{M}=\mathfrak{A}_{N}$.

The assumption in the corollary certainly implies that every real has a sharp, and from this and the fact that $M$ is essentially countable, it follows that $V_{\delta+1}^{M}$ is countable in $V$ (indeed, $\omega_{1}^{V}$ is a inaccessible in $L\left(V_{\delta}^{M}\right)$ which contains $\left.V_{\delta+1}^{M}\right)$. This allows us to use Lemmas 4.3 and 4.7 .

If $n=0$, then using Lemma 4.7 we obtain an essentially countable, almost fine structural, $\Pi_{2}^{1}$ iterable model $N^{*}$ with a Woodin cardinal, so that $\mathfrak{A}_{N}=\mathfrak{A}_{M}$ embeds elementarily into $\mathfrak{A}_{N^{*}}$, and so that $\alpha$ is a countable ordinal in $N^{*}$. The fact that $\alpha$ is countable in $N^{*}$ allows constructing a code $w$ for $\alpha$ inside $N^{*}$, and this establishes the corollary.

Suppose $n \geq 1$. Using Lemma 4.3 we obtain an essentially countable, almost fine structural, $\Pi_{2}^{1}$ iterable model $N^{*}$ with a Woodin cardinal, so that $\mathfrak{A}_{N}=\mathfrak{A}_{M}$ embeds elementarily into $\mathfrak{A}_{N^{*}}$, and so that there is a real $v \in N^{*}$ with $v_{u_{n}}^{+}>\alpha$. (If $n=1$ this is a direct application of the lemma. If $n>1$, we use also the fact that $u_{n}$, for $1 \leq n<\omega$, are the uniform indiscernibles, and therefore there is some $\bar{\alpha} \in\left[u_{1}, u_{2}\right)$ so that $v_{\omega_{1}}^{+}>\bar{\alpha}$ implies $v_{u_{n}}^{+}>\alpha$. We then apply the lemma to $\bar{\alpha}$.)

Since $v_{u_{n}}^{+}>\alpha$, there is some ordinal $\beta<u_{n}$ so that $\alpha$ is $\Sigma_{1}$ definable in $L(v)$ from $\beta$ and $u_{n}$. By induction, there is $N^{* *}$ so that $\mathfrak{A}_{N^{*}}$ embeds elementarily into $\mathfrak{A}_{N^{* *}}$, and $\beta$ has a code $w$ in $N^{* *}$. The fact that $\mathfrak{A}_{N^{*}}$ embeds elementarily into $\mathfrak{A}_{N^{* *}}$ implies that $v \in N^{* *}$. From $v$ and the code $w$ for $\beta$ one can create a code for $\alpha$. This code belongs to $N^{* *}$.

§5. The theorems. Recall that $\alpha_{x}$, for $x \in \mathbb{R}$, is the least ordinal so that $L_{\alpha_{x}}\left(T_{2}, x\right)$ is admissible. For an essentially countable model $N$ and a real $x \in N$, $L_{\gamma_{x}^{N}}\left(T_{N}, x\right)$ is an initial approximation of $L_{\alpha_{x}}\left(T_{2}, x\right)$ in $N . T_{N}$ is defined by letting $Z_{N}$ be the set of ordinals below $u_{\omega}$ with codes in $N$, letting $c_{N}: Z_{N} \rightarrow X_{N}$ be the collapse embedding, and setting $T_{N}$ to be the image of $T_{2}$ under $c_{N}$. Let $\eta_{N}$ denote $\sup \left(X_{N}\right)$, so that $T_{N}$ is a tree on $\omega \times \eta_{N}$. 
$\gamma_{x}^{N}$ is the least ordinal so that $L_{\gamma_{x}^{N}}\left(T_{N}, x\right)$ is admissible. This ordinal may be too large for the purpose of approximating $L_{\alpha_{x}}\left(T_{2}, x\right)$. The correct ordinal is given by the next definition.

Definition 5.1. Define $\delta_{x}^{N}$ to be the least ordinal $\delta<\gamma_{x}^{N}$ for which there is $\vec{a} \in \eta_{N}^{<\omega}$ and a $\Sigma_{1}$ formula $\psi$ so that $L_{\delta+1}\left(T_{N}, x\right) \models \psi\left(T_{N}, x, \vec{a}\right)$ but $L_{\alpha_{x}}\left(T_{2}, x\right) \not \models$ $\psi\left(T_{2}, x, c_{N}^{-1}(\vec{a})\right)$, if such an ordinal exists. If there is no such ordinal, set $\delta_{x}^{N}=\gamma_{x}^{N}$.

We will use the structure $L_{\delta_{x}^{N}}\left(T_{N}, x\right)$ as an approximation for $L_{\alpha_{x}}\left(T_{2}, x\right)$. We show, in Lemma 5.2, that there is a $\Sigma_{1}$ elementary embedding of the approximation into the original structure. We then use the approximation to prove the Kechris-Martin theorem and its corollary.

We work throughout the section in a universe satisfying ZF + DC plus the existence of $M_{1}^{\sharp}(x)$, the fully iterable inner model with the sharp of one Woodin cardinal over $x$, for each real $x$, or equivalently $\boldsymbol{\Delta}_{2}^{1}$ determinacy.

Throughout this section, a model is called nice if it is essentially countable, almost fine structural, $\Pi_{2}^{1}$ iterable, with a Woodin cardinal. In other words a model is nice if it is pre-nice in the sense of Section 4 and $\Pi_{2}^{1}$ iterable.

Lemma 5.2. Let $N$ be nice. Then there is a $\Sigma_{1}$ elementary embedding $\pi$ of $L_{\delta_{x}^{N}}\left(T_{N}, x\right)$ into $L_{\alpha_{x}}\left(T_{2}, x\right)$.

Moreover $\pi$ is determined completely by the following conditions:

1. For $\xi<\eta_{N}, \pi(\xi)=c_{N}^{-1}(\xi)$.

2. For every $\xi \in\left[\eta_{N}, \delta_{x}^{N}\right)$, every $\vec{a} \in \eta_{N}^{<\omega}$, and every $\Sigma_{1}$ formula $\psi$ so that $\xi$ is least for which $L_{\xi+1}\left(T_{N}, x\right) \models \psi\left(T_{N}, x, \vec{a}\right), \pi(\xi)$ is the least ordinal so that $L_{\pi(\xi)+1}\left(T_{2}, x\right) \models \psi\left(T_{2}, x, \pi(\vec{a})\right)$.

(These conditions determine the restriction of $\pi$ to ordinals, and this in turn trivially determines all other values of $\pi$.)

Proof. Let $g: \omega \rightarrow \mathbb{R}$ be generic for $\operatorname{col}(\omega, \mathbb{R})$ over $V$. Working in $V[g]$, using repeated applications of Corollary 4.8 in $V$, construct a sequence of models $N_{i}$, $i<\omega$, all in $V$, and all nice in $V$, so that:

1. $N_{0}=N$.

2. $\mathfrak{A}_{N_{i}}$ embeds elementarily into $\mathfrak{A}_{N_{i+1}}$.

3. If $g(i)$ is a code for an ordinal below $u_{\omega}$, then there is a code for the same ordinal, inside $N_{i+1}$.

Using condition (2), fix for each $i$ an elementary embedding $\sigma_{i}: \mathfrak{A}_{N_{i}} \rightarrow \mathfrak{A}_{N_{i+1}}$. Let $\pi_{i, i+1}$ be the restriction of $\sigma_{i}$ to $L_{\gamma_{x}^{N_{i}}}\left(T_{N_{i}}, x\right)$. Then using the properties of the structures $\mathfrak{A}_{N_{i}}$ in Remark 4.2, $\pi_{i, i+1}: L_{\gamma_{x}^{N_{i}}}\left(T_{N_{i}}, x\right) \rightarrow L_{\gamma_{x}^{N_{i+1}}}\left(T_{N_{i+1}}, x\right)$ is elementary, and for each code $w \in N_{i}, \pi_{i, i+1}\left(c_{N_{i}}(|w|)\right)=c_{N_{i+1}}(|w|)$.

Let $Q$ be the direct limit of the structures $L_{\gamma_{x}^{N_{i}}}\left(T_{N_{i}}, x\right)$ under the embeddings $\pi_{i, i+1}$ and their compositions. Let $\pi_{i, \infty}: L_{\gamma_{x}^{N_{i}}}\left(T_{N_{i}}, x\right) \rightarrow Q$ be the direct limit embeddings.

By condition (3) and the genericity of $g$, all ordinals below $u_{\omega}$ have codes in $\bigcup_{i<\omega} N_{i}$. Using this and the fact that $\pi_{i, j}\left(c_{N_{i}}(|w|)\right)=c_{N_{j}}(|w|)$, it is easy to check that $\pi_{i, \infty}\left(c_{N_{i}}(|w|)\right)=|w|$ for each code $w$ in $N_{i}$. It follows from this and the definition of the trees $T_{N_{i}}$ that $\pi_{i, \infty}\left(T_{N_{i}}\right)$ is the tree $T_{2}$, and hence $Q$ satisfies 
" $V$ is the least admissible structure containing $T_{2}$ and $x "$. This implies that $Q$ is an end extension of the model $L_{\alpha_{x}}\left(T_{2}, x\right)$. $Q$ can be a proper end extension, but only if it is illfounded, in which case its wellfounded part is equal to $L_{\alpha_{x}}\left(T_{2}, x\right)$.

Since $\gamma_{x}^{N}$ is least so that $L_{\gamma_{x}^{N}}\left(T_{N}, x\right)$ is admissible, for every ordinal $\xi \in$ $\left[\eta_{N}, \gamma_{x}^{N}\right)$ there is some $\vec{a} \in \eta_{N}^{<\omega}$ and some $\Sigma_{1}$ formula $\psi$ so that $\xi$ is least for which $L_{\xi+1}\left(T_{N}, x\right) \models \psi\left(T_{N}, x, \vec{a}\right)$. If $\xi<\delta_{x}^{N}$, then by definition of $\delta_{x}^{N}$ there must exist some $\xi^{*}$ below $\alpha_{x}$, so that $L_{\xi^{*}+1}\left(T_{2}, x\right) \models \psi\left(T_{2}, x, \pi_{0, \infty}(\vec{a})\right)$, and the least such $\xi^{*}$ must be equal to $\pi_{0, \infty}(\xi)$, by elementarity of $\pi_{0, \infty}$. If follows that the restriction of $\pi_{0, \infty}$ to $L_{\delta_{x}^{N}}\left(T_{N}, x\right)$ is an embedding of $L_{\delta_{x}^{N}}\left(T_{N}, x\right)$ into $L_{\alpha_{x}}\left(T_{2}, x\right)$, and that it is determined in the manner specified in the lemma. It remains to show that the embedding is $\Sigma_{1}$ elementary.

Let $\theta$ be a $\Sigma_{1}$ formula in parameters $a_{1}^{*}, \ldots, a_{k}^{*}$ that belong to the range of $\pi_{0, \infty}\left\lceil L_{\delta_{x}^{N}}\left(T_{N}, x\right)\right.$. We may without loss of generality assume that $a_{1}^{*}, \ldots, a_{k}^{*}$ are ordinals. We may also assume that they belong to the image of $\eta_{N}^{<\omega}$, since we have seen in the previous paragraph that all ordinals in the range of $\pi_{0, \infty}\left\lceil L_{\delta_{x}^{N}}\left(T_{N}, x\right)\right.$ are $\Sigma_{1}$ definable from ordinals in the image of $\eta_{N}^{<\omega}$ and that $\pi_{0, \infty}$ respects these definitions. Fix $a_{1}, \ldots, a_{k} \in \eta_{N}$ so that $a_{i}^{*}=\pi_{0, \infty}\left(a_{i}\right)$.

Suppose that $\theta\left(T_{2}, x, a_{1}^{*}, \ldots, a_{k}^{*}\right)$ holds in $L_{\alpha_{x}}\left(T_{2}, x\right)$. We have to prove that $\theta\left(T_{N}, x, a_{1}, \ldots, a_{k}\right)$ holds in $L_{\delta_{x}^{N}}\left(T_{N}, x\right)$.

Suppose first that $\delta_{x}^{N}=\gamma_{x}^{N}$. Since $Q$ extends $L_{\alpha_{x}}\left(T_{2}, x\right)$, and $\theta$ is $\Sigma_{1}$, $\theta\left(T_{2}, x, a_{1}^{*}, \ldots, a_{k}^{*}\right)$ holds in $Q$. Hence by elementarity of $\pi_{0, \infty}, L_{\gamma_{x}^{N}}\left(T_{N}, x\right)=$ $L_{\delta_{x}^{N}}\left(T_{N}, x\right)$ satisfies $\theta\left(T_{N}, x, a_{1}, \ldots, a_{k}\right)$.

Suppose next that $\delta_{x}^{N}<\gamma_{x}^{N}$. Then since $\pi_{0, \infty}$ is elementary into $Q$, it follows from the definition of $\delta_{x}^{N}$ that $\pi_{0, \infty}\left(\delta_{x}^{N}\right)$ cannot belong to $L_{\alpha_{x}}\left(T_{2}, x\right)$, and must belong instead to the illfounded part of $Q$. Since $\theta$ is $\Sigma_{1}$, this in turn implies that $\theta\left(T_{2}, x, a_{1}^{*}, \ldots, a_{k}^{*}\right)$ holds in $\left(L_{\pi_{0, \infty}\left(\delta_{x}^{N}\right)}\left(T_{2}, x\right)\right)^{Q}$. (This model is an end extension of $L_{\alpha_{x}}\left(T_{2}, x\right)$.) By elementarity of $\pi_{0, \infty}$, it follows that $\theta\left(T_{N}, x, a_{1}, \ldots, a_{k}\right)$ holds in $L_{\delta_{x}^{N}}\left(T_{N}, x\right)$.

The proof of Lemma 5.2 yields the following additional claims that help characterize $\delta_{x}^{N}$.

Claim 5.3. Let $N$ be nice, and let $x, y$ be reals in $N$. Suppose that $\delta_{x}^{N}<\gamma_{x}^{N}$. Then $\delta_{y}^{N} \leq \delta_{x}^{N}$.

Proof. Suppose for contradiction that $\delta_{x}^{N}<\delta_{y}^{N}$. Let $\psi_{x}$ and $\vec{a}$ be as in the definition of $\delta_{x}^{N}$. Let $z$ be a real in $N$ that codes the pair $\langle x, y\rangle$. Then $\gamma_{z}^{N} \geq \max \left\{\gamma_{x}^{N}, \gamma_{y}^{N}\right\}$, hence $\delta_{x}^{N}<\gamma_{z}^{N}$.

Let $\pi: L_{\gamma_{z}^{N}}\left(T_{N}, z\right) \rightarrow Q$ be the map $\pi_{0, \infty}$ defined in the proof of Lemma 5.2 , applied for the real $z$.

Since $\delta_{x}^{N}<\delta_{y}^{N} \leq \gamma_{y}^{N}$, there is some $\Sigma_{1}$ formula $\theta$, with parameters in $\eta_{N}^{<\omega}$, which without loss of generality we may assume are contained in $\vec{a}$, so that $\delta_{x}^{N}$ is least so that $L_{\delta_{x}^{N}+1}\left(T_{N}, y\right) \models \theta\left(T_{N}, y, \vec{a}\right)$. The model $L_{\delta_{x}^{N}+1}\left(T_{N}, z\right)$ then satisfies " $L\left(T_{N}, x\right)$ is a model of $\psi_{x}\left(T_{N}, x, \vec{a}\right), L\left(T_{N}, x\right)$ is not admissible and has no admissible initial segments containing $T_{N}$ and $x$, and no strict initial segment of $L\left(T_{N}, y\right)$ is a model of $\theta\left(T_{N}, y, \vec{a}\right)$ ".

By elementarity of $\pi,\left(L_{\pi\left(\delta_{x}^{N}+1\right)}\left(T_{2}, z\right)\right)^{Q}$ satisfies the same formula with $T_{N}$ replaced by $T_{2}$ and $\vec{a}$ replaced by $\pi(\vec{a})=c_{N}^{-1}(\vec{a})$. From the final clause in 
this formula, the fact that $Q$ is an end extension of $L_{\alpha_{z}}\left(T_{2}, z\right)$ which contains $L_{\alpha_{y}}\left(T_{2}, y\right)$, and the assumption that $\delta_{x}^{N}<\delta_{y}^{N}$, which by definition of $\delta_{y}^{N}$ implies that $\theta$ holds at some level of $L_{\alpha_{y}}\left(T_{2}, y\right)$, it follows that $\pi\left(\delta_{x}^{N}+1\right)$ must be smaller than $\alpha_{y}$.

But then $\pi\left(\delta_{x}^{N}+1\right)$ is smaller than $\alpha_{z}$, and in particular it belongs to the wellfounded part of $Q$. From this and the other parts of the formula above, it follows that $L_{\pi\left(\delta_{x}^{N}+1\right)}\left(T_{2}, x\right)$ is a strict initial segment of $L_{\alpha_{x}}\left(T_{2}, x\right)$, and satisfies $\psi_{x}\left(T_{2}, x, c_{N}^{-1}(\vec{a})\right)$. This contradicts the definition of $\delta_{x}^{N}$.

Recall that $\gamma_{N}=\sup \left\{\gamma_{x}^{N} \mid x \in N\right\}$. If there exists some $x \in N$ so that $\delta_{x}^{N}<\gamma_{x}^{N}$, define $\delta_{N}$ to be $\min \left\{\delta_{x}^{N} \mid x \in N\right.$ and $\left.\delta_{x}^{N}<\gamma_{x}^{N}\right\}$. If no such $x$ exists, set $\delta_{N}=\gamma_{N}$. By Claim 5.3 we then immediately get:

Claim 5.4. For every real $y \in N, \delta_{y}^{N}=\min \left\{\gamma_{y}^{N}, \delta_{N}\right\}$.

Proof. If $\delta_{N}=\gamma_{N}$ then $\delta_{y}^{N}=\gamma_{y}^{N}$ for all $y$ and there is nothing further to prove. Suppose $\delta_{N}<\gamma_{N}$ and let $x$ realize the minimum in the definition of $\delta_{N}$. By Claim 5.3, $\delta_{y}^{N} \leq \delta_{x}^{N}$. Since $\delta_{y}^{N} \leq \gamma_{y}^{N}$ by definition, it follows that $\delta_{y}^{N} \leq$ $\min \left\{\gamma_{y}^{N}, \delta_{N}\right\}$. If $\delta_{y}^{N}<\gamma_{y}^{N}$, then applying the Claim 5.3 with $x$ and $y$ reversed it follows that $\delta_{N}=\delta_{x}^{N} \leq \delta_{y}^{N}$. Hence $\delta_{y}^{N}<\min \left\{\gamma_{y}^{N}, \delta_{N}\right\}$ is impossible.

CLAIM 5.5. $\delta_{N}<\gamma_{N}$ iff there exists a sequence $\left\langle N_{i}, \alpha_{i}, \sigma_{i} \mid i<\omega\right\rangle$ so that:

1. $N_{0}=N$.

2. Each $N_{i}$ is nice (meaning essentially countable, almost fine structural, with a Woodin cardinal, and $\Pi_{2}^{1}$ iterable).

3. $\alpha_{i}<\gamma_{N_{i}}$.

4. $\sigma_{i}$ is an elementary embedding of $\mathfrak{A}_{N_{i}}$ into $\mathfrak{A}_{N_{i+1}}$.

5. $\alpha_{i+1}<\sigma_{i}\left(\alpha_{i}\right)$.

Moreover, $\delta_{N}$ is the least $\alpha_{0}$ for which such a sequence exists.

Proof. Suppose first that there is a sequence $\left\langle N_{i}, \alpha_{i}, \sigma_{i} \mid i<\omega\right\rangle$ satisfying conditions (1)-(5). Fix $x \in N$ so that $\alpha_{0}<\gamma_{x}^{N}$. Such an $x$ exists since $\alpha_{0}<\gamma_{N}$. Note that condition (4) implies in particular that $x \in N_{i}$ for each $i$.

Since $N_{i}$ is nice, Lemma 5.2 applies to it. Let $\pi_{i}: L_{\delta_{x}^{N_{i}}}\left(T_{N_{i}}, x\right) \rightarrow L_{\alpha_{x}}\left(T_{2}, x\right)$ be the embedding given by the lemma.

By conditions (2), the elementarity of $\sigma_{i}$, and the properties of $\mathfrak{A}_{N_{i}}$ in Remark $4.2, \sigma_{i}\left(c_{N_{i}}(|w|)\right)=c_{N_{i+1}}(|w|)$ for each code $w$ in $N_{i}$. From this, the elementarity of the maps $\sigma_{i}$, and the characterization of the embedding in Lemma 5.2, it follows that the maps $\pi_{i}$ and $\sigma_{i}$ commute. Precisely, $\pi_{i}(\xi)=\left(\pi_{i+1} \circ \sigma_{i}\right)(\xi)$ for every $\xi<\delta_{x}^{N_{i}}$.

If $\alpha_{0}$ belongs to the domain of $\pi_{0}$, then by condition (5) together with the commutativity in the previous paragraph, $\left\langle\pi_{i}\left(\alpha_{i}\right) \mid i<\omega\right\rangle$ forms an infinite descending chain in $L_{\alpha_{x}}\left(T_{2}, x\right)$. Since this is impossible, $\alpha_{0}$ must be outside the domain of $\pi_{0}$. This implies by definitions that $\delta_{x}^{N} \leq \alpha_{0}$. In particular $\delta_{x}^{N}<\gamma_{x}^{N}$, hence $\delta_{N}<\gamma_{N}$, and indeed $\delta_{N} \leq \alpha_{0}$.

We have seen so far that the existence of a sequence satisfying conditions (1)(5) implies that $\delta_{N}<\gamma_{N}$ and $\delta_{N} \leq \alpha_{0}$. It remains to show that, assuming $\delta_{N}<\gamma_{N}$, there is a sequence satisfying these conditions, with $\alpha_{0}=\delta_{N}$. 
Suppose $\delta_{N}<\gamma_{N}$, and let $x$ be a real realizing the minimum in the definition of $\delta_{N}$, meaning that $\delta_{x}^{N}<\gamma_{x}^{N}$ and $\delta_{N}=\delta_{x}^{N}$. Let $R$ be the tree of attempts to construct a sequence satisfying conditions (1)-(5), with $\alpha_{0}=\delta_{N}=\delta_{x}^{N}$. We must show that $R$ is not wellfounded. By absoluteness of wellfoundedness it is enough to show that there is a branch through $R$ in some generic extension of the universe.

Let $g$ be generic for $\operatorname{col}(\omega, \mathbb{R})$. Let $\left\langle N_{i}, \sigma_{i}\right| i\langle\omega\rangle \in V[g]$ be the sequence constructed in the proof of Lemma 5.2. This sequence satisfies conditions (1), (2), and (4). Since $\delta_{N}=\delta_{x}^{N}<\gamma_{x}^{N}$, the last paragraph in the proof of Lemma 5.2 shows that $\pi_{0, \infty}\left(\delta_{x}^{N}\right)$ belongs to the illfounded part of $Q$. Hence there is a sequence of ordinals $\alpha_{i}, i<\omega$, so that $\alpha_{0}=\delta_{N}=\delta_{x}^{N}$ and so that $\alpha_{i+1}<$ $\pi_{i, i+1}\left(\alpha_{i}\right)=\sigma\left(\alpha_{i}\right)$ for each $i$. The combined sequence $\left\langle N_{i}, \alpha_{i}, \sigma_{i} \mid i<\omega\right\rangle$ then satisfies conditions (1)-(5).

We have now accumulated enough information to identify pairs $\langle N, \mu\rangle$ so that $N$ is nice and $\mu \in\left[\delta_{N}, \gamma_{N}\right]$, in a $\Sigma_{3}^{1}$ manner. Let $Z$ be the set of pairs $\langle N, \mu\rangle$ so that:

1. $N$ is essentially countable, almost fine structural, with a Woodin cardinal.

2. $N$ is $\Pi_{2}^{1}$ iterable.

3. $\mu \leq \gamma_{N}$.

4. Either $\mu=\gamma_{N}$, or else there exists a sequence satisfying conditions (1)-(5) of Claim 5.5, with $\alpha_{0} \leq \mu$.

Claim 5.6. The set $Z$ is $\Sigma_{3}^{1}$, and $\langle N, \mu\rangle \in Z$ iff $N$ is nice and $\mu \in\left[\delta_{N}, \gamma_{N}\right]$.

Proof. The fact that $\langle N, \mu\rangle \in Z$ iff $N$ is nice and $\mu \in\left[\delta_{N}, \gamma_{N}\right]$ is immediate from the definitions and Claim 5.5. The definition of $Z$ involves quantifiers asserting the existence of models, ordinals, and embeddings with certain properties. These properties are all first order, except for being an essentially countable model with a Woodin cardinal and $\Pi_{2}^{1}$ iterability, which are both $\Pi_{2}^{1}$. It follows that $Z$ is $\Sigma_{3}^{1}$.

TheOREm 5.7. Let $\varphi$ be a $\Pi_{1}$ formula. Then the set $\left\{x \mid L_{\alpha_{x}}\left(T_{2}, x\right) \models\right.$ $\left.\varphi\left(T_{2}, x\right)\right\}$ is $\Sigma_{3}^{1}$.

Proof. By Lemma 5.2 and since $\varphi$ is $\Pi_{1}$, for every nice $N$ with $x \in N$, $L_{\alpha_{x}}\left(T_{2}, x\right) \models \varphi\left(T_{2}, x\right)$ iff $L_{\delta_{x}^{N}}\left(T_{N}, x\right) \models \varphi\left(T_{N}, x\right)$. By Claim 5.4, and again using the fact that $\varphi$ is $\Pi_{1}, L_{\delta_{x}^{N}}\left(T_{N}, x\right) \models \varphi\left(T_{N}, x\right)$ in turn is equivalent to the existence of $\mu \geq \delta_{N}$ so that $L_{\min \left\{\gamma_{x}^{N}, \mu\right\}}\left(T_{N}, x\right) \models \varphi\left(T_{N}, x\right)$.

We are assuming throughout the section that over every real $x$ there is a fully iterable model for the sharp of a Woodin cardinal, and this implies in particular that there exist nice $N$ with $x \in N$. Hence, by the previous paragraph and Claim $5.6, L_{\alpha_{x}}\left(T_{2}, x\right) \models \varphi\left(T_{2}, x\right)$ iff there is $\langle N, \mu\rangle \in Z$ so that $L_{\min \left\{\gamma_{x}^{N}, \mu\right\}}\left(T_{N}, x\right) \models$ $\varphi\left(T_{N}, x\right)$. Since $Z$ is $\Sigma_{3}^{1}$, and the clause $L_{\min \left\{\gamma_{x}^{N}, \mu\right\}}\left(T_{N}, x\right) \models \varphi\left(T_{N}, x\right)$ is first order over $N$, this is a $\Sigma_{3}^{1}$ statement.

We phrased Theorem 5.7 for $\Pi_{1}$ formulas and $\Sigma_{3}^{1}$ sets, but of course it is equivalent to the assertion that $\left\{x \mid L_{\alpha_{x}}\left(T_{2}, x\right) \models \varphi\left(T_{2}, x\right)\right\}$ is $\Pi_{3}^{1}$ when $\varphi$ is $\Sigma_{1}$, in other words to Corollary 1.4.

The Kechris-Martin theorem itself can also be proved using inner models, as follows. Again for convenience we phrase it for $\Sigma_{3}^{1}$ rather than $\Pi_{3}^{1}$. 
THEOREM 5.8. $\Sigma_{3}^{1}$ is closed under universal quantification over ordinals below $u_{\omega}$.

Proof. The only property of $T_{2}$ that we have used so far is Fact 4.1. This was sufficient for the proof of Theorem 5.7. But now we use also the fact that $T_{2}$ projects to a universal $\Pi_{2}^{1}$ set. This immediately implies the converse of Theorem 5.7, namely that for every $\Sigma_{3}^{1}$ set $A$, there is a $\Pi_{1}$ formula $\varphi$ so that $A=\left\{x \mid L_{\alpha_{x}}\left(T_{2}, x\right) \models \varphi\left(T_{2}, x\right)\right\}$. (The formula states that there is no embedding of the tree of attempts to witness $x \in A$, into the ordinals. A witness for $x \in A$ includes a real and a branch through $T_{2}$, together certifying a $\Sigma_{3}^{1}$ statement.)

Let $A$ be $\Sigma_{3}^{1}$, with elements of the form $\langle x, w\rangle$ where $x$ is a real and $w$ a code for an ordinal below $u_{\omega}$. Suppose that for every $x$, if $\left|w_{1}\right|=\left|w_{2}\right|$ then $\left\langle x, w_{1}\right\rangle \in A \Longleftrightarrow\left\langle x, w_{2}\right\rangle \in A$. We prove that the set $B=\{x \mid(\forall w)\langle x, w\rangle \in A\}$ is $\Sigma_{3}^{1}$.

Fix a $\Pi_{1}$ formula $\varphi$ so that $\langle x, w\rangle \in A$ iff $L_{\alpha\langle x, w\rangle}\left(T_{2}, x, w\right) \models \varphi\left(T_{2}, x, w\right)$. It is enough to prove that $x \in B$ iff there exists $\langle N, \mu\rangle \in Z$ so that for every code $w \in N, L_{\min \left\{\gamma_{x, w}^{N}, \mu\right\}}\left(T_{N}, x, w\right) \models \varphi\left(T_{N}, x, w\right)$. This is a $\Sigma_{3}^{1}$ statement, since $Z$ is $\Sigma_{3}^{1}$, and since all other quantifiers in the statement are over elements of $N$.

The left-to-right direction of the equivalence is clear, using the existence of a nice (in fact fully iterable) model $N$ with $x \in N$, Theorem 5.2, Claim 5.6, and Claim 5.4. We prove the right-to-left direction.

Suppose $\langle N, \mu\rangle \in Z$, and for every code $w \in N, L_{\min \left\{\gamma_{x, w}^{N}, \mu\right\}}\left(T_{N}, x, w\right) \models$ $\varphi\left(T_{N}, x, w\right)$. Fix an ordinal $\alpha<u_{\omega}$. By Corollary 4.8 , there is a nice $N^{*}$ so that there is a code $w^{*}$ for $\alpha$ in $N^{*}$, and so that $\mathfrak{A}_{N}$ embeds elementarily into $\mathfrak{A}_{N^{*}}$. Let $\sigma$ be the embedding. By elementarity of $\sigma$ then, and using the properties of $\mathfrak{A}_{N}$ and $\mathfrak{A}_{N^{*}}$ given by condition (4) of Remark 4.2, $L_{\min \left\{\gamma_{x, w^{*}}^{N^{*}}, \sigma(\mu)\right\}}\left(T_{N^{*}}, x, w^{*}\right) \models$ $\varphi\left(T_{N^{*}}, x, w^{*}\right)$ (where $\sigma(\mu)$ is understood to mean $\gamma_{N^{*}}$ if $\mu=\gamma_{N}$ ).

It is enough now to show that $\sigma(\mu) \geq \delta_{N^{*}}$, since then by Theorem 5.2 and Claim 5.4 it follows that $\left.L_{\alpha_{x, w^{*}}}\left(T_{2}, x, w^{*}\right) \models \varphi\left(T_{2}, x, w^{*}\right)\right\}$, so $\left\langle x, w^{*}\right\rangle \in A$ as required.

If $\delta_{N}=\gamma_{N}$ then $\mu=\gamma_{N}, \sigma(\mu)=\gamma_{N^{*}} \geq \delta_{N^{*}}$, and there is nothing further to prove. Suppose $\delta_{N}<\gamma_{N}$. Let $y \in N$ realize the minimum in the definition of $\delta_{N}$, so that $\delta_{N}=\delta_{y}^{N}<\gamma_{y}^{N}$. Let $\psi$ and $\vec{a}$ be as in the definition of $\delta_{y}^{N}$, so that in particular $L_{\delta_{N}+1}\left(T_{N}, y\right) \models \psi\left(T_{N}, y, \vec{a}\right)$. By the elementarity of $\sigma$ and Remark 4.2, $L_{\sigma\left(\delta_{N}\right)+1}\left(T_{N^{*}}, y\right) \models \psi\left(T_{N^{*}}, y, \sigma(\vec{a})\right)$. If $\sigma\left(\delta_{N}\right)<\delta_{N^{*}}$ then using Lemma 5.2 it follows that $L_{\alpha_{y}}\left(T_{2}, y\right) \models \psi\left(T_{2}, y, c_{N}^{-1}(\vec{a})\right)$, which contradicts the definition of $\psi_{y}$. So $\sigma\left(\delta_{N}\right) \geq \delta_{N^{*}}$. Now since $\mu \geq \delta_{N}, \sigma(\mu) \geq \delta_{N^{*}}$.

THEOREM 5.9. Every non-empty $\Pi_{3}^{1}$ set of ordinals below $u_{\omega}$ has a $\Delta_{3}^{1}$ element.

Proof. Let $B$ be a non-empty $\Pi_{3}^{1}$ set of ordinals below $u_{\omega}$. Let $\psi$ be a $\Sigma_{1}$ formula so that $|w| \in B$ iff $L_{\alpha_{w}}\left(T_{2}, w\right) \models \psi\left(T_{2}, w\right)$. Strengthening $\psi$ if necessary we may assume that $\psi\left(T_{2}, w\right)$ implies that there are no admissible sets containing $T_{2}$ and $w$. Let $\beta$ be least so that $L_{\beta+1}\left(T_{2}, w\right) \models \psi\left(T_{2}, w\right)$ for some $w$. Then $L_{\beta+1}\left(T_{2}, w\right) \models \psi\left(T_{2}, w\right)$ implies $\beta<\alpha_{w}$ and $|w| \in B$.

Let $A$ be the complement of $B$. The proof of Theorem 5.8, applied to the $\Sigma_{3}^{1}$ set $\{0\} \times A$, shows that for any nice $N$, if all ordinals with codes in $N$ belong 
to $A$, then in fact all ordinals below $u_{\omega}$ belong to $A$. Rephrasing, if $B$ has no elements with codes in $N$, then it is empty. Since $B$ is non-empty it follows that every nice $N$ has codes for elements of $B$. In other words, every nice $N$ must have codes $w$ so that $L_{\alpha_{w}}\left(T_{2}, w\right) \models \psi\left(T_{2}, w\right)$.

For each nice $N$ which is fully fine structural (including below $\omega_{1}^{N}$ ), let $\beta_{N}$ be least so that $L_{\beta_{N}+1}\left(T_{N}, w\right) \models \psi\left(T_{N}, w\right)$ for some $w$ in $N$, and let $w_{N}$ be the least (in the canonical enumeration of the reals in $N$ ) code $w$ witnessing this. By Lemma 5.2 and since there is $w \in N$ so that $L_{\alpha_{w}}\left(T_{2}, w\right) \models \psi\left(T_{2}, w\right)$, an ordinal $\beta_{N}$ satisfying this definition exists in $N$, and moreover it is smaller than $\delta_{N}$. Since $\psi\left(T_{N}, w\right)$ cannot hold in levels containing an admissible set over $T_{N}$ and $w, \beta_{N}$ must also be smaller than $\gamma_{w_{N}}^{N}$, and hence smaller than $\gamma_{x}^{N}$ for every real $x \in N$ which computes $w_{N}$.

It follows that for every such $x, \beta_{N}<\delta_{x}^{N}$, and $L_{\pi\left(\beta_{N}\right)+1}\left(T_{2}, w_{N}\right) \models \psi\left(T_{2}, w_{N}\right)$, where $\pi: L_{\delta_{x}^{N}}\left(T_{N}, x\right) \rightarrow L_{\alpha_{x}}\left(T_{2}, x\right)$ is the embedding given by Lemma 5.2. In particular, by the minimality of $\beta, \pi\left(\beta_{N}\right) \geq \beta$.

Let $B^{\prime}$ be the set of codes $w$ for which $\left(\exists \eta<\alpha_{\left\langle w, w_{N}\right\rangle}\right)$ so that $L_{\eta+1}\left(T_{2}, w\right) \models$ $\psi\left(T_{2}, w\right)$ and $L_{\eta+1}\left(T_{2}, w_{N}\right) \not \forall \psi\left(T_{2}, w_{N}\right)$. The minimality of $\beta_{N}$ implies that $B^{\prime}$ has no elements in $N$. Since $B^{\prime}$ is $\Pi_{3}^{1}$ in $w_{N}$, a relativized version of the argument in the first two paragraph of this proof shows that $B^{\prime}$ has no elements at all. This in turn implies that $\pi\left(\beta_{N}\right) \leq \beta$, and hence $\pi\left(\beta_{N}\right)=\beta$.

Taking $x=w_{N}$ it follows that $L_{\beta+1}\left(T_{2}, w_{N}\right) \models \psi\left(T_{2}, w_{N}\right)$. Moreover, $w_{N}$ is the least code $w \in N$ (least in the order of the reals of $N$ ) so that $L_{\beta+1}\left(T_{2}, w\right) \models$ $\psi\left(T_{2}, w\right)$. For if there is a smaller $w \in N$ with this property, taking $x$ which computes both $w$ and $w_{N}$ and using the fact that $\pi\left(\beta_{N}\right)=\beta$ we get $L_{\beta_{N}+1}\left(T_{N}, w\right) \models$ $\psi\left(T_{N}, w\right)$, contradicting the minimality in the definition of $w_{N}$.

A comparison argument similar to that in the proof of Lemma 2.2 shows that the reals of $M_{1}^{\sharp}$, the minimal fully iterable model for the sharp of one Woodin cardinal, form an initial segment of the reals of any fully fine structural nice $N$, where in the context of a fully fine structural model, the $\Pi_{2}^{1}$ iterability given by niceness applies to iteration trees using any extenders on the sequence of the model, including also extenders indexed below $\omega_{1}^{N}$. Since $w_{N}$ is the least code $w \in N$ so that $L_{\beta+1}\left(T_{2}, w\right) \models \psi\left(T_{2}, w\right)$, and since such a code exists in $M_{1}^{\sharp}$ (as it does in every nice model), $w_{N}$ must be equal to $w_{M_{1}^{\sharp}}$. This holds for every fully fine structural nice $N$, and allows characterizing $w_{M_{1}^{\sharp}}$, which codes an element of $B$, in a $\Delta_{3}^{1}$ way. Precisely, $w=w_{M_{1}^{\sharp}}$ iff the following equivalent conditions hold:

1. There exists a fully fine structural, essentially countable, $\Pi_{2}^{1}$ iterable $N$ with a Woodin cardinal, so that letting $\beta_{N} \in N$ be least so that $(\exists v \in$ $N) L_{\beta_{N}+1} \models \psi\left(T_{N}, v\right), w$ is equal to the least witness $v$ for this, in the ordering of the reals of $N$.

2. For every $N$ as in (1), and every $\beta_{N}$ which is least so that $(\exists v \in N) L_{\beta_{N}+1} \models$ $\psi\left(T_{N}, v\right), w$ is equal to the least witness $v$ for this, in the ordering of the reals of $N$.

Condition (1) is $\Sigma_{3}^{1}$, and condition (2) is $\Pi_{3}^{1}$. 


\section{REFERENCES}

[1] A. S. Kechris and D. A. Martin, On the theory of $\Pi_{3}^{1}$ sets of reals, Bull. Amer. Math. Soc., vol. 84 (1978), no. 1, pp. 149-151.

[2] D. A. Martin and J. R. Steel, Iteration trees, J. Amer. Math. Soc., vol. 7 (1994), no. 1 , pp. $1-73$.

[3] Itay Neeman, Optimal proofs of determinacy, Bull. Symbolic Logic, vol. 1 (1995), no. 3 , pp. 327-339. 258.

[4] —, Optimal proofs of determinacy. II, J. Math. Log., vol. 2 (2002), no. 2, pp. 227-

[5] John R. Steel, An outline of inner model theory, Handbook of set theory. Vols. 1, 2, 3, Springer, Dordrecht, 2010, pp. 1595-1684.

DEPARTMENT OF MATHEMATICS

UNIVERSITY OF CALIFORNIA LOS ANGELES

LOS ANGELES, CA 90095-1555

E-mail: ineeman@math.ucla.edu 